\title{
Heterospirocyclic 3-Amino-2H-azirines as Convenient Building Blocks in Peptide Synthesis
}

\author{
Strässler, Christoph ; Heimgartner, Heinz
}

\begin{abstract}
Heterospirocyclic 3-amino-2H-azirines with a tetrahydropyran (Thp), tetrahydrothiopyran (Tht), or N-protected piperidine (Pip) moiety are useful reagents for the preparation of peptides containing the corresponding six-membered heterocyclic 4-amino-4-carboxylic acid unit. In the present study, tripeptides of the type H-Asp-D-Ala-Xaa-OMe, where Xaa is the heterocyclic amino acid, were prepared according to the 'azirine/oxazolone method'.
\end{abstract}

DOI: https://doi.org/10.3987/COM-18-S(F)41

Posted at the Zurich Open Repository and Archive, University of Zurich

ZORA URL: https://doi.org/10.5167/uzh-157432

Journal Article

Accepted Version

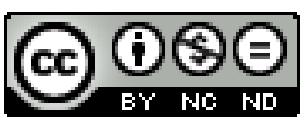

The following work is licensed under a Creative Commons: Attribution-NonCommercial-NoDerivatives 4.0 International (CC BY-NC-ND 4.0) License.

Originally published at:

Strässler, Christoph; Heimgartner, Heinz (2019). Heterospirocyclic 3-Amino-2H-azirines as Convenient Building Blocks in Peptide Synthesis. Heterocycles, 99(1):446.

DOI: https://doi.org/10.3987/COM-18-S(F)41 


\title{
HETEROCYCLES, Vol. 99, No. 1, 2019, pp. 446 - 464. @ 2019 The Japan Institute of Heterocyclic Chemistry Received, 24th August, 2018, Accepted, 13th September, 2018, Published online, 24th October, 2018 DOI: $10.3987 / C O M-18-S(F) 41$ \\ HETEROSPIROCYCLIC 3-AMINO-2H-AZIRINES AS CONVENIENT BUILDING BLOCKS IN PEPTIDE SYNTHESIS
}

\section{Christoph Strässler ${ }^{1}$ and Heinz Heimgartner*}

Department of Chemistry, University of Zurich, Winterthurerstrasse 190, CH-8057 Zurich, Switzerland. E-mail: heinz.heimgartner@chem.uzh.ch

Dedicated to Professor Dr. Tohru Fukuyama on the occasion of his 70th birthday

\begin{abstract}
Heterospirocyclic 3-amino-2H-azirines with a tetrahydropyran (Thp), tetrahydrothiopyran (Tht), or $N$-protected piperidine (Pip) moiety are useful reagents for the preparation of peptides containing the corresponding six-membered heterocyclic 4-amino-4-carboxylic acid unit. In the present study, tripeptides of the type H-Asp-D-Ala-Xaa-OMe, where Xaa is the heterocyclic amino acid, were prepared according to the 'azirine/oxazolone method'.
\end{abstract}

\section{INTRODUCTION}

The so-called 'azirine/oxazolone method'2 is a convenient approach for the synthesis of peptides containing $\alpha, \alpha$-disubstituted $\alpha$-amino acids, e.g., $\alpha$-aminoisobutyric acid (peptaibols). ${ }^{3}$ The utility of the 2,2-dimethyl-substituted 3-amino- $2 \mathrm{H}$-azirine $\mathbf{1 a}$ in the preparation of such peptides has been demonstrated in a long series of publications. ${ }^{4}$ A special type of $\alpha, \alpha$-dialkylated $\alpha$-amino acids are fiveand six-membered heterocyclic n-amino-n-carboxylic acids, ${ }^{5,6}$ and their synthons in the method mentioned above are spirocyclic 3-amino- $2 H$-azirines $\mathbf{1 b}-\mathbf{e}^{7}$

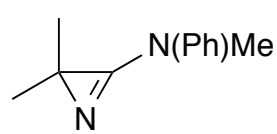

1a

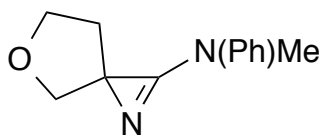

$1 b$

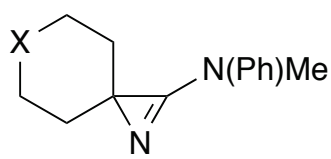

$1 c X=$ NBoc

$1 d x=0$

$1 e x=S$

Figure 1. 3-Amino- $2 H$-azirines as synthons for $\alpha$-aminoisobutyric acid (Aib, 1a) and heterocyclic n-amino-n-carboxylic acids (1b-e) 
In a recent publication, we have shown that 3 -amino- $2 H$-azirines $\mathbf{1 c}-\mathbf{e}$ are useful synthons for the preparation of tripeptides of type 4 (Scheme 1), which can be used further for the synthesis of larger peptides. ${ }^{8}$ The first reaction step is the azirine coupling with an $N$-protected amino acid $\mathbf{2}$, followed by a selective hydrolysis of the terminal amide function and the second coupling with an amino acid ester $\mathbf{3}$ via a 1,3-oxazol-5-one intermediate.

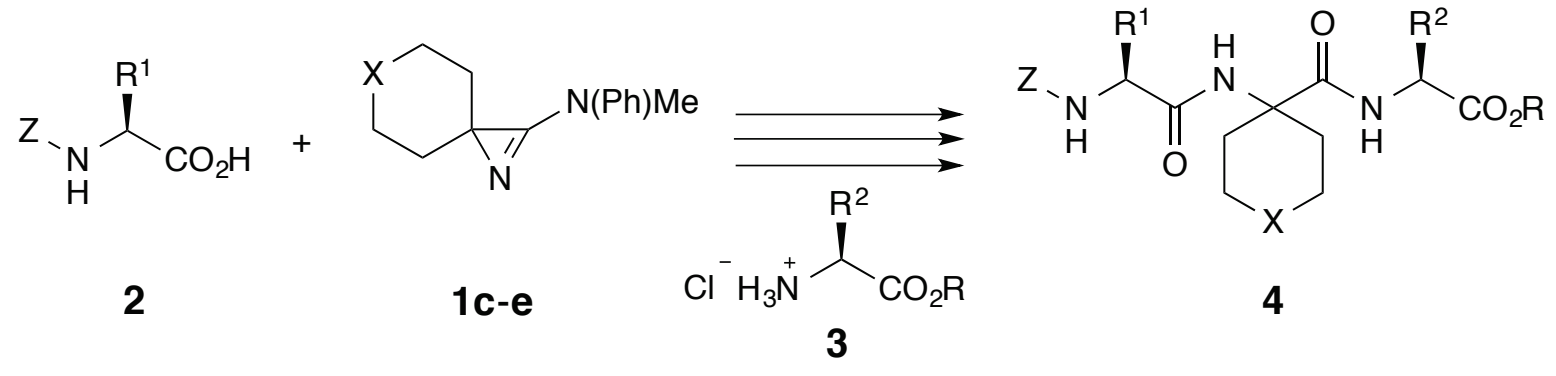

Scheme 1. Synthesis of tripeptides of type 4 ( $Z$ = benzyloxycarbonyl)

An interesting type of tripeptides containing $\alpha, \alpha$-disubstituted $\alpha$-amino acids are L-aspartyl-D-alanyl- $\mathrm{AC}_{\mathrm{n}} \mathrm{C}-\mathrm{OMe}$ derivatives, with $\mathrm{AC}_{\mathrm{n}} \mathrm{C}=1$-aminocycloalkane-1-carboxylic acids, which have been studied as potential peptide sweeteners by Goodman and coworkers. ${ }^{9}$ For example, it was shown that the taste of the tripeptides with the six-membered and smaller carbocyclic amino acids is sweet, whereas the analogues with larger carbocyclic rings taste bitter. The goal of the present study was the synthesis of analogous tripeptides containing a heterocyclic six-membered 4-amino-4-carboxylic acid by using heterospirocyclic 3 -amino- $2 \mathrm{H}$-azirines of type $\mathbf{1 c - e}$ as amino acid synthons.

\section{RESULTS AND DISCUSSION}

Whereas the syntheses of the heterospirocyclic 3-amino- $2 \mathrm{H}$-azirines $\mathbf{1 c}-\mathbf{e}$ were carried out under standard conditions, ${ }^{7 \mathrm{c}}$ the preparation of analogues of $\mathbf{1 c}$ with a benzyloxycarbonyl (Z) or allyloxycarbonyl (Alloc) protecting group failed. With the intention to have in hand also a synthon for 4-aminopiperidine-4-carboxylic acid with a benzyloxycarbonyl-type of protecting group, we prepared the 3',5'-dimethoxy-1,1-dimethylbenzyloxycarbonyl (Ddz)-protected azirine 1f (Scheme 2). Thus, the reaction of the azide $5^{10}$ with $N$-methyl- $N$-phenylpiperidine-4-carboxamide hydrochloride (6) in $\mathrm{CH}_{2} \mathrm{Cl}_{2} / \mathrm{Et}_{3} \mathrm{~N}$ at room temperature gave the Ddz-protected amide 7 in $96 \%$ yield. Stepwise reaction of the latter with LDA, diphenyl chlorophosphate (DPPCl), and $\mathrm{NaN}_{3}$ according to our previously described protocol $^{7 \mathrm{~b}, 11}$ led to the desired azirine $\mathbf{1 f}$ in $76 \%$ yield. 
<smiles>COc1cc(OC)cc(C(C)(C)OC(N)=O)c1</smiles>

5<smiles>O=C(Nc1ccccc1)C1CCNCC1</smiles>

6

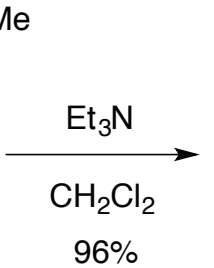

96\%

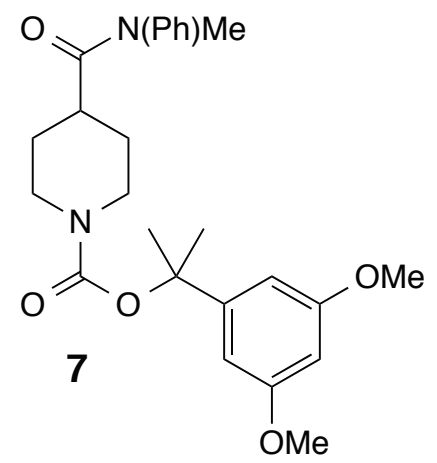

1) LDA, THF, $0^{\circ} \mathrm{C}, 2 \mathrm{~h}$

2) $\mathrm{DPPCl}, \mathrm{THF}, 0^{\circ} \mathrm{C}$ to rt, $24 \mathrm{~h}$

3) $\mathrm{NaN}_{3}, \mathrm{THF}, \mathrm{rt}, 3 \mathrm{~d}$

$76 \%$<smiles>COc1cc(OC)cc(C(C)(C)C)c1</smiles>

$1 f$

Scheme 2. Synthesis of the Ddz-protected heterospirocyclic 3-amino- $2 H$-azirine $\mathbf{1 f}$

Similar to other $2 \mathrm{H}$-azirines of type $\mathbf{1}$, the new derivative $\mathbf{1 f}$ is a stable compound, which can be stored in the refrigerator for long time. Furthermore, its reactivity towards acidic compounds is analogous to that of 1a-e. For example, the reaction with thiobenzoic acid in $\mathrm{CH}_{2} \mathrm{Cl}_{2}$ at room temperature ${ }^{11}$ gave the 4-(benzoylamino)piperidine-4-thioamide 8 in $79 \%$ yield (Scheme 3).

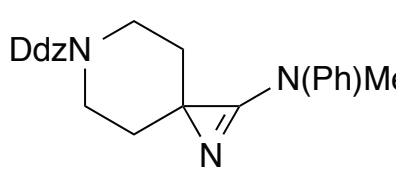

$1 f$<smiles>CCCN(C)C(=O)C1(NC(=O)c2ccccc2)CCN(C(C)C)CC1</smiles>

$8(79 \%)$

Scheme 3. Reaction of 3-amino- $2 H$-azirine 1 f with thiobenzoic acid

The reaction of the heterospirocyclic 3-amino- $2 \mathrm{H}$-azirines 1d-f with $\mathrm{Z}$ - or Fmoc-protected D-alanine (D-Ala) under the standard conditions of the 'azirine coupling' ( $\mathrm{MeCN}, \mathrm{rt}$ ) led to the enantiomerically pure (NMR) dipeptide amides 10a-d in 85-95\% yield (Scheme 4, Table 1). In analogy to the selective hydrolysis of peptide amides of type 10, treatment of $\mathbf{1 0 a}, \mathbf{b}$ with $\mathrm{HCl}$ gas in $\mathrm{MeOH}$ at $\mathrm{rt}$ to ca. $60{ }^{\circ} \mathrm{C}$ gave the dipeptide methyl esters 11a,b in excellent yields without loss of stereochemical purity. In the case of the piperidine derivative 10c, the Ddz-protecting group was also removed, and the methyl ester 11c with the free $\mathrm{NH}$ group in the heterocycle was obtained in $68 \%$ yield. Subsequent deprotection of the terminal amino group of 11a,b by hydrogenolysis or treatment with piperidine in $\mathrm{CH}_{2} \mathrm{Cl}_{2}$, respectively, yielded the desired dipeptide methyl esters $\mathbf{1 2 a}, \mathbf{b}$ in good yields. 
<smiles>[X]C1CCC2(CC1)NC2[NH3+]</smiles>

1d-f $X=O, S, N(D d z)$<smiles>C[C@H](NP)C(=O)O</smiles>

9a,b $P=Z, F m o c$<smiles>[X]CCC(C)(C)NC(=O)[C@H](C)NC(=O)[C@H](C)NP</smiles>

$10 a-d$

$\mathrm{HCl}(\mathrm{g})$

$\mathrm{MeOH}, \mathrm{rt}$

$\mathrm{H}_{2}, \mathrm{Pd} / \mathrm{C}, \mathrm{MeOH}$<smiles>[X]CCC(CC)(NC(=O)[C@H](C)N)C(=O)OC</smiles>

$12 a, b$ or

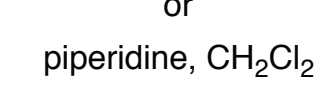

11a-c

Scheme 4. Synthesis of dipeptide esters 12 via 'azirine coupling' (see also Table 1)

Table 1. Synthesis of H-Asp-D-Ala-Xaa-OMe tripeptides 15 via the 'azirine/oxazolone method'

\begin{tabular}{|c|c|c|c|c|c|c|c|c|c|c|c|c|c|}
\hline $\mathbf{1}$ & $\mathrm{X}$ & $\mathbf{9}$ & $\mathrm{P}$ & $\mathbf{1 0}$ & $\begin{array}{c}\text { Yield } \\
(\%)^{\mathrm{a})}\end{array}$ & $\mathbf{1 1}$ & $\begin{array}{c}\text { Yield } \\
(\%)^{\mathrm{a})}\end{array}$ & $\mathbf{1 2}$ & $\begin{array}{c}\text { Yield } \\
(\%)^{\mathrm{a})}\end{array}$ & $\mathbf{1 4}$ & $\begin{array}{c}\text { Yield } \\
(\%)^{\mathrm{a})}\end{array}$ & $\mathbf{1 5}$ & $\begin{array}{c}\text { Yield } \\
(\%)^{\mathrm{a})}\end{array}$ \\
\hline $\mathbf{d}$ & $\mathrm{O}$ & $\mathbf{a}$ & $\mathrm{Z}$ & $\mathbf{a}$ & 92 & $\mathbf{a}$ & 92 & $\mathbf{a}$ & 81 & $\mathbf{a}$ & 80 & $\mathbf{a}$ & 98 \\
$\mathbf{e}$ & $\mathrm{S}$ & $\mathbf{b}$ & $\mathrm{Fmoc}$ & $\mathbf{b}$ & 87 & $\mathbf{b}$ & 94 & $\mathbf{b}$ & 76 & $\mathbf{b}$ & 92 & $\mathbf{b}$ & ca. $\left.90^{\mathrm{j}}\right)$ \\
$\mathbf{f}$ & $\mathrm{N}(\mathrm{Ddz})$ & $\mathbf{a}$ & $\mathrm{Z}$ & $\mathbf{c}$ & 95 & $\left.\mathbf{c}^{\mathrm{b}}\right)$ & 68 & & & - & & - & \\
& & & & & $\left.\mathbf{d}^{\mathrm{c}}\right)$ & 87 & & & - & & - & \\
& & & & & $\left.\mathbf{e}^{\mathrm{d}}\right)$ & 70 & - & & $\left.\mathbf{c}^{\mathrm{f}}\right)$ & 84 & $\left.\mathbf{c}^{\mathrm{h}}\right)$ & quant. \\
$\mathbf{f}$ & $\mathrm{N}(\mathrm{Ddz})$ & $\mathbf{b}$ & Fmoc & $\mathbf{d}$ & 85 & $\left.\mathbf{f}^{\mathbf{e}}\right)$ & 76 & - & & $\left.\mathbf{d}^{\mathrm{g}}\right)$ & 44 & $\left.\mathbf{d}^{\mathrm{i}}\right)$ & ca. $\left.90^{\mathrm{j}}\right)$ \\
\hline
\end{tabular}

$\left.{ }^{a}\right)$ Yield of isolated product. $\left.{ }^{b}\right)$ The dipeptide ester with the deprotected piperidine ring $(\mathrm{X}=\mathrm{NH})$ was obtained. $\left.^{\mathrm{c}}\right) \mathrm{X}=\mathrm{N}($ Fmoc $), \mathrm{P}=\mathrm{Z}$; obtained from 11c. $\left.{ }^{\mathrm{d}}\right) \mathrm{X}=\mathrm{N}($ Boc $), \mathrm{P}=\mathrm{Z}$; obtained from 11d. $\left.{ }^{\mathrm{e}}\right) \mathrm{X}=$ $\mathrm{N}(\mathrm{Z}), \mathrm{P}=$ Fmoc; obtained from 10d. $\left.\left.{ }^{f}\right) \mathrm{X}=\mathrm{N}(\mathrm{Boc}), \mathrm{P}^{1}=\mathrm{Z}, \mathrm{P}^{2}=\mathrm{Bn} .{ }^{\mathrm{g}}\right) \mathrm{X}=\mathrm{N}(\mathrm{Z}), \mathrm{P}^{1}=$ Fmoc, $\mathrm{P}^{2}=t \mathrm{Bu}$. ${ }^{\text {h) }} \mathrm{X}=\mathrm{N}($ Boc $\left.) .{ }^{\mathrm{i}}\right) \mathrm{X}=\mathrm{N}(Z) .{ }^{\mathrm{j}}$ ) Not obtained in pure form.

With the aim of obtaining dipeptide methyl esters of type 11 with a $N$-protected piperidine moiety, 11c was treated with Fmoc-chloride and $\mathrm{Et}_{3} \mathrm{~N}$ in $\mathrm{CH}_{2} \mathrm{Cl}_{2}$ at $\mathrm{rt}$ to give Z-D-Ala-Pip(Fmoc)-OMe (11d) in $87 \%$ yield (Table 1). The same product could be prepared from 10c in an analogous manner without isolation of the intermediate 11c (76\% yield). Furthermore, the exchange of the Fmoc against the Boc protecting group was performed by subsequent treatment of $\mathbf{1 1 d}$ with piperidine in $\mathrm{CH}_{2} \mathrm{Cl}_{2}$ followed by 
Boc-anhydride $\left.(\mathrm{Boc})_{2} \mathrm{O}\right) / \mathrm{Et}_{3} \mathrm{~N}$ to give Z-D-Ala-Pip(Boc)-OMe (11e). Similarly, Fmoc-D-Ala-Pip(Z)-OMe (11f) was obtained from 10d via acid-catalyzed methanolysis and treatment of the crude product with $\mathrm{Et}_{3} \mathrm{~N}$ and Z-chloride (76\% yield).

Unfortunately, the isolated deprotected dipeptides 12a,b were not soluble in suitable solvents for peptide coupling. For this reason, the coupling with the diprotected aspartic acid derivatives Z-Asp(OBn)-OH (13a) and Fmoc-Asp $(\mathrm{O} t \mathrm{Bu})-\mathrm{OH}(\mathbf{1 3 b})$, respectively, were carried out with the crude materials 12a,b. Thus, 11a was deprotected hydrogenolytically in $\mathrm{DMF} / \mathrm{MeOH}$, the catalyst was removed by filtration and $\mathrm{MeOH}$ evaporated. Then, 13a, $\mathrm{Et}_{3} \mathrm{~N}$, and $\mathrm{PyBOP}$ as the coupling reagent were added, and after $16 \mathrm{~h}$ the tripeptide 14a was obtained in $80 \%$ yield (Scheme 5, Table 1). In an analogous manner, 11e was coupled with 13a to give the fully protected Z-Asp(OBn)-D-Ala-Pip(Boc)-OMe (14c) in 84\% yield. Both tripeptides were transformed into the desired zwitterionic analogues 15a,c via hydrogenolytic deprotection.
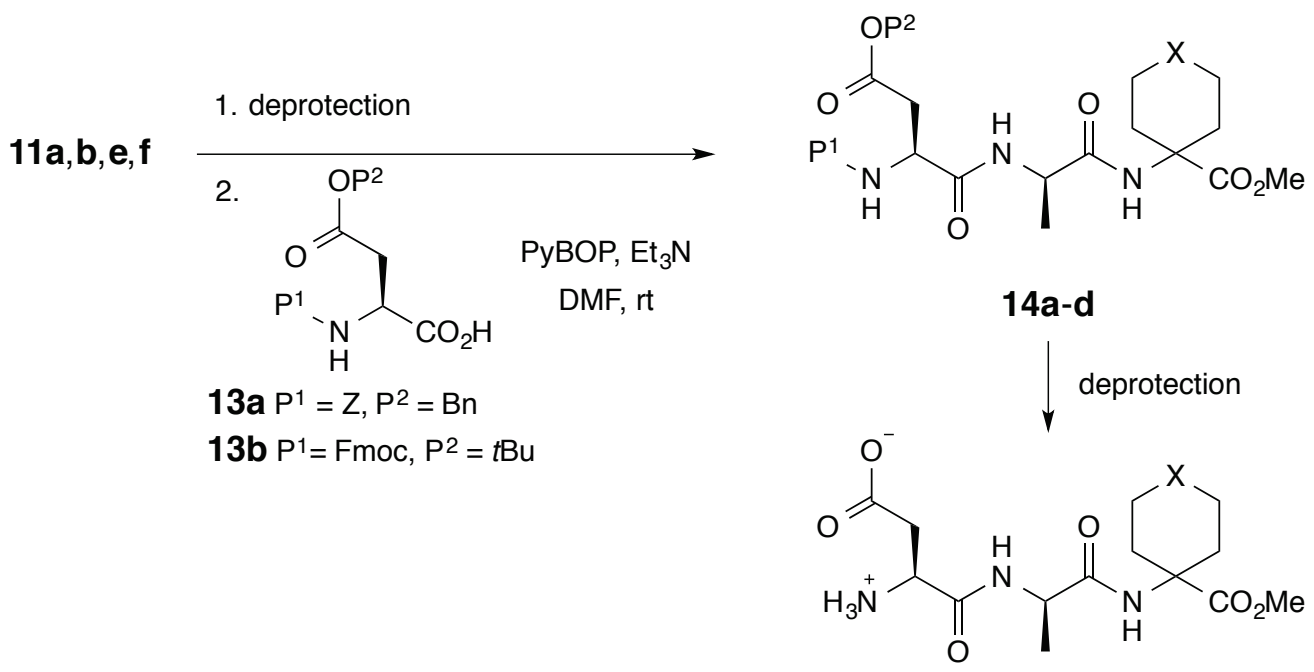

$15 a-d$

Scheme 5. Synthesis of tripeptide methyl esters 15 (H-Asp-D-Ala-Xaa-OMe; see also Table 1)

The Fmoc-protected dipeptides $\mathbf{1 1 b}, \mathbf{f}$ were deprotected by treatment with piperidine in DMSO at rt. After evaporation of piperidine, 13b and PyBOP were added, and after stirring at $\mathrm{rt}$ for $16 \mathrm{~h}$ and chromatographic purification, 14b and 14d were isolated in 92 and 44\% yield, respectively. The deprotection to give $\mathbf{1 5 b}$,d was achieved in two steps: removal of the Fmoc group with piperidine in $\mathrm{CH}_{2} \mathrm{Cl}_{2}$ at $\mathrm{rt}$ and subsequent cleavage of the tert-butyl ester by treatment with trifluoroacetic acid (TFA). Whereas the partially deprotected tripeptides H-Asp $(\mathrm{O} t \mathrm{Bu})$-D-Ala-Tht-OMe and $\mathrm{H}-\mathrm{Asp}(\mathrm{O} t \mathrm{Bu})-\mathrm{D}-\mathrm{Ala}-\mathrm{Pip}(\mathrm{Z})-\mathrm{OMe}$ were obtained in pure forms, the desired zwitterionic compounds $\mathbf{1 5 b} \mathbf{b}$ were still contaminated with small amounts of impurities, which could not be removed. 


\section{CONCLUSIONS}

The heterospirocyclic 3-amino- $2 H$-azirines $\mathbf{1 d}-\mathbf{f}$ were shown to be convenient starting materials for the synthesis of peptides containing saturated six-membered heterocyclic 4-amino-4-carboxylic acids. In addition to the previously described $\mathbf{1 d}$ and 1e containing a tetrahydropyran and tetrahydrothiopyran ring, respectively, the new synthon 1f, a 1,6-diazaspiro[2.5]oct-1-ene derivative with a Ddz-protected piperidine moiety, was used for the 'azirine coupling' with $N$-protected D-alanine to give the corresponding dipeptide amides in high yields. The Ddz protecting group can be removed easily under acidic conditions allowing the smooth exchange of the protecting group. The terminal amide function of the dipeptides with a heterocyclic $\alpha$-amino carboxylic acid was transformed into the corresponding methyl ester via selective methanolysis. Deprotection of the $N$-terminus and coupling with asparagine derivatives leads to the fully protected tripeptide esters of type $\mathrm{P}^{1}-\mathrm{Asp}\left(\mathrm{P}^{2}\right)$-D-Ala-Xaa-OMe. The deprotection of the examples with hydrogenolytically removable groups was achieved successfully to give the zwitterionic tripeptide esters, which are analogues of those with a carbocyclic $\alpha$-amino carboxylic acid. It is worth mentioning that the taste of tripeptide 15a with a tetrahydropyran moiety is sweet, similar to the cyclohexane analogue, ${ }^{9}$ whereas that of $\mathbf{1 5 c}$ with a Boc-protected piperidine ring is bitter.

\section{EXPERIMENTAL}

General remarks. Melting points were determined using a Mettler FP5 apparatus, and they are uncorrected. Thin layer chromatography (TLC): Merck silica gel $60 \mathrm{~F}_{254}$ plates $(0.25 \mathrm{~mm})$; column chromatography (CC): silica gel Merck $60(0.040-0.063 \mathrm{~mm}$. The IR spectra were recorded on a Perkin-Elmer 297 or Perkin-Elmer 781 spectrophotometer in $\mathrm{CDCl}_{3}$ or in $\mathrm{KBr}$; absorptions in $\mathrm{cm}^{-1}$. The ${ }^{1} \mathrm{H}$ - and ${ }^{13} \mathrm{C}-\mathrm{NMR}$ spectra were measured on a Bruker ARX-300, AM-400 or AMX-600 instrument (300/75.4, 400/100.6, and 600/150.9 $\mathrm{MHz}$, resp.) in $\mathrm{CDCl}_{3}$ with TMS as internal standard. Chemical shifts $(\delta)$ are given in ppm and coupling constants $J$ in Hz. Mass spectra (MS) were recorded on a Finnigan SSQ-700 (CI, $\left.\mathrm{NH}_{3}, 150 \mathrm{eV}\right)$ or Finnigan TSQ-700 (ESI) instrument. Optical rotations $\left[\alpha_{\mathrm{D}}\right]$ : Zeiss LEP-A 2 polarimeter, in $\mathrm{MeOH}$ at $20-22^{\circ} \mathrm{C}$.

Starting materials. The synthesis of $N$-methyl- $N$-phenyl-6-oxa-1-azaspiro[2.5]oct-1-en-2-amine (1d) and $N$-methyl- $N$-phenyl-6-thia-1-azaspiro[2.5] oct-1-en-2-amine (1e) has been described previously. ${ }^{7 \mathrm{~b}}$ The amino acid derivatives Z- and Fmoc-D-Ala $(\mathbf{9 a}, \mathbf{b})$ and Z, Bn- and Fmoc, $t$ Bu-protected Asp as well as all used reagents were commercially available. Reported yields refer to isolated pure products. 
Abbreviations. AcOEt, ethyl acetate; D-Ala, D-alanine; Alloc, allyloxycarbonyl; Asp, aspartic acid; Bn, benzyl; Boc, tert-butyloxycarbonyl; CC, column chromatography; Ddz, 3',5'-dimethoxy-1,1-dimethylbenzyloxycarbonyl; DMF, N,N-dimethylformamide; DPPCl, diphenyl chlorophosphate; $\mathrm{Et}_{2} \mathrm{O}$, diethyl ether; Fmoc, fluorenylmethyloxycarbonyl; LDA, lithium diisopropylamide; MeCN, acetonitrile; Pip, 4-aminopiperidine-4-carboxylic acid; PyBOP, [(benzotriazol-1-yl)oxy]tripyrrolidinophosphonium hexafluorophosphate; THF, tetrahydrofuran; Thp, 4-aminotetrahydropyran-4-carboxylic acid; Tht, 4-aminotetrahydrothiopyran-4-carboxylic acid; Z, benzyloxycarbonyl.

\section{Synthesis of 6-[(3',5'-dimethoxy-1,1-dimethylbenzyloxy)carbonyl]-2-( $N$-methyl- $N$-phenylamino)-} 1,6-diazaspiro[2.5]oct-1-ene (1f). 1-[(3',5'-Dimethoxy-1,1-dimethylbenzyloxy)carbonyl]piperidine-4( $N$-methyl- $N$-phenylcarboxamide) $\quad$ (7). To a stirred solution $\quad$ of 9.86 g $\quad\left(\begin{array}{llll}38.7 & \mathrm{mmol}\end{array}\right)$ piperidine-4-( $N$-methyl- $N$-phenylcarboxamide) hydrochloride $(6)$ in $\mathrm{CH}_{2} \mathrm{Cl}_{2}(100 \mathrm{~mL})$ at $0{ }^{\circ} \mathrm{C}$ were added $\mathrm{Et}_{3} \mathrm{~N}(12 \mathrm{~mL}, 86 \mathrm{mmol})$ and (3',5'-dimethoxy-1,1-dimethylbenzyloxy)carbonyl azide ${ }^{10}$ (5; $11.3 \mathrm{~g}, 42.6$ mmol). After $1 \mathrm{~h}$ at $\mathrm{rt}$, the mixture was washed with aqueous $\mathrm{Na}_{2} \mathrm{CO}_{3}$ solution $(10 \%, 2 \times)$, and the aqueous solutions were extracted with $\mathrm{CH}_{2} \mathrm{Cl}_{2}$. The combined organic phases were dried $\left(\mathrm{Na}_{2} \mathrm{SO}_{4}\right)$ and the solvent evaporated. $\mathrm{CC}$ (hexane/AcOEt 1:2) and recrystallization from $\mathrm{CH}_{2} \mathrm{Cl}_{2}$ gave $16.5 \mathrm{~g}$ (96\%) of 7. Colorless crystals; mp $125-126{ }^{\circ} \mathrm{C}$. IR $\left(\mathrm{CHCl}_{3}\right): 3460 w, 3020 w, 3000 m, 2950 m, 2860 w, 2840 w, 1680 s, 1645 s$, $1600 \mathrm{~s}, 1495 \mathrm{~m}, 1470 \mathrm{~m}, 1455 \mathrm{~m}, 1430 \mathrm{~s}, 1390 \mathrm{~m}, 1350 \mathrm{~m}, 1310 \mathrm{~m}, 1300 \mathrm{~m}, 1285 \mathrm{~m}, 1270 \mathrm{~m}, 1220 \mathrm{~m}, 1160 \mathrm{~s}$, $1135 m, 1070 m, 1050 m, 1025 m, 965 w, 935 w, 890 w, 845 w, 835 w .{ }^{1} \mathrm{H}-\mathrm{NMR}\left(\left(\mathrm{D}_{6}\right) \mathrm{DMSO}, 85{ }^{\circ} \mathrm{C}\right)$ : 7.47-7.26, 6.44-6.43, 6.36-6.34 (3m, 8 arom. H); 3.97-3.68 (dt-like, $2 \mathrm{H}$ of $\left.-\mathrm{CH}_{2} \mathrm{NCH}_{2}-\right)$; $3.73(s, 2$ $\mathrm{MeO}) ; 3.16(s, \mathrm{MeN}) ; 2.62-2.40\left(m, 2 \mathrm{H}\right.$ of $\left.-\mathrm{CH}_{2} \mathrm{NCH}_{2}-, \mathrm{CH}(4)\right) ; 1.66\left(s, \mathrm{Me}_{2} \mathrm{C}\right) ; 1.61-1.44(m$, $\left.-\mathrm{CH}_{2} \mathrm{CCH}_{2}-\right) .{ }^{13} \mathrm{C}-\mathrm{NMR}\left(\left(\mathrm{D}_{6}\right) \mathrm{DMSO}, 85^{\circ} \mathrm{C}\right): 173.1$ ( $\left.s, \mathrm{C}=\mathrm{O}\right) ; 160.6$ (s, 2 arom. COMe); 152.6, 148.4, 143.5 (3s, OCON, 2 arom. C); 129.1, 126.9, 126.6, 102.6, 98.2 (5d, 8 arom. CH); 79.9 ( $\left.s, \mathrm{Me}_{2} \mathrm{CO}\right) ; 54.7$ $(q, 2 \mathrm{MeO}) ; 42.4\left(t,-\mathrm{CH}_{2} \mathrm{NCH}_{2}-\right) ; 37.8(d, \mathrm{CH}(4)) ; 36.6(q, \mathrm{MeN}) ; 28.2\left(q, M e_{2} \mathrm{C}\right) ; 27.7\left(t,-\mathrm{CH}_{2} \mathrm{CCH}_{2}-\right)$. ESI-MS: $463\left(100,[M+\mathrm{Na}]^{+}\right), 241$ (36). Anal. Calcd for $\mathrm{C}_{25} \mathrm{H}_{32} \mathrm{~N}_{2} \mathrm{O}_{5}$ (440.54): C 68.16, H 7.32, N 6.36. Found: C 68.18, H 7.32, N 6.40.

\section{6-[(3',5'-Dimethoxy-1,1-dimethylbenzyloxy)carbonyl]-2-( $N$-methyl- $N$-phenylamino)-1,6-diazaspiro-}

[2.5]oct-1-ene (1f). To a solution of $7(7.00 \mathrm{~g}, 15.9 \mathrm{mmol})$ in THF $(40 \mathrm{~mL})$ at $0{ }^{\circ} \mathrm{C}$ under $\mathrm{Ar}$ atmosphere was added a $1.5 \mathrm{M}$ solution of LDA in cyclohexane $(9.5 \mathrm{~mL}, 19 \mathrm{mmol})$, and the mixture was stirred at $0{ }^{\circ} \mathrm{C}$ for $90 \mathrm{~min}$. Then, DPPCl $(4.72 \mathrm{~g}, 17.6 \mathrm{mmol})$ was added by means of a syringe at $0{ }^{\circ} \mathrm{C}$, the solution stirred at $0{ }^{\circ} \mathrm{C}$ for $30 \mathrm{~min}$ and at $\mathrm{rt}$ for $24 \mathrm{~h}$. The formed precipitate was filtered of under Ar atmosphere, the filtrate was added to $3.12 \mathrm{~g}(47.9 \mathrm{mmol}) \mathrm{NaN}_{3}$ and the mixture stirred at $\mathrm{rt}$ for $3 \mathrm{~d}$. After addition of 
$\mathrm{Et}_{2} \mathrm{O}$ and filtration of the mixture on Celite, the solvents of the filtrate were evaporated, the residue dissolved in $\mathrm{CH}_{2} \mathrm{Cl}_{2}$ and washed with aqueous $\mathrm{NaHCO}_{3}(5 \%, 3 \times)$. The combined aqueous phases were washed with $\mathrm{CH}_{2} \mathrm{Cl}_{2}$, the combined organic phases were dried $\left(\mathrm{MgSO}_{4}\right)$, and the solvent evaporated. Purification via $\mathrm{CC}\left(\mathrm{SiO}_{2}\right.$, hexane/AcOEt 2:1) gave $5.31 \mathrm{~g} \mathrm{(76 \% )} \mathrm{of} \mathrm{the} \mathrm{azirine} \mathrm{1f.} \mathrm{Colorless,} \mathrm{viscous} \mathrm{oil.}$ IR $\left(\mathrm{CHCl}_{3}\right): 3000 m, 2970 m, 2940 m, 2860 w, 2840 w, 1750 s, 1685 s, 1600 s, 1500 m, 1460 s, 1425 s, 1380 w$, $1365 m, 1350 m, 1320 m, 1300 m, 1275 m, 1250 m, 1155 s, 1100 s, 1070 m, 1020 m, 960 w, 890 w, 835 w, 695 w$, 610w. ${ }^{1} \mathrm{H}-\mathrm{NMR}\left(\left(\mathrm{D}_{6}\right) \mathrm{DMSO}, 85{ }^{\circ} \mathrm{C}\right)$ : 7.45-7.33, 7.16-7.10, 6.52-6.51, 6.41-6.39 (4m, 8 arom. H); $3.78-3.70\left(m, 2 \mathrm{H}\right.$ of $\left.-\mathrm{CH}_{2} \mathrm{NCH}_{2}-\right) ; 3.76(s, 2 \mathrm{MeO}) ; 3.52-3.42\left(m, 2 \mathrm{H}\right.$ of $\left.-\mathrm{CH}_{2} \mathrm{NCH}_{2}-\right)$; $3.43(s, \mathrm{MeN})$; 1.95-1.84 ( $m, 2 \mathrm{H}$ of $\left.-\mathrm{CH}_{2} \mathrm{CCH}_{2}-\right) ; 1.74\left(s, \mathrm{Me}_{2} \mathrm{C}\right) ; 1.42-1.34\left(m, 2 \mathrm{H}\right.$ of $\left.-\mathrm{CH}_{2} \mathrm{CCH}_{2}-\right) .{ }^{13} \mathrm{C}-\mathrm{NMR}$ $\left(\left(\mathrm{D}_{6}\right) \mathrm{DMSO}, 85{ }^{\circ} \mathrm{C}\right): 165.7,160.0,152.9,148.8,142.1$ (5s, C(2), 2 arom. COMe, OCON, 2 arom. C); 128.7, 122.7, 116.8, 102.7, 98.1 (5d, 8 arom. $\mathrm{CH})$; 80.1 ( $\left.s, \mathrm{Me}_{2} C \mathrm{O}\right) ; 54.7$ ( $\left.q, 2 \mathrm{MeO}\right) ; 42.5(t$, $\left.-\mathrm{CH}_{2} \mathrm{NCH}_{2}-\right) ; 35.1(q, \mathrm{MeN}) ; 34.2\left(t,-\mathrm{CH}_{2} \mathrm{CCH}_{2}-\right) ; 28.2\left(q, M e_{2} \mathrm{C}\right) ; \mathrm{C}(3)$ could not be detected. ESI-MS: $439(28), 438\left(100,[M+1]^{+}\right), 260(34), 179(25)$.

\section{Reaction of azirine 1f with thiobenzoic acid. Synthesis of $N$-\{1-[(3',5'-dimethoxy-1,1-dimethyl-} benzyloxy)carbonyl]-4-( $N$-methyl- $N$-phenylthiocarbamoyl)piperidin-4-yl\}benzamide $\quad(8)$. To a solution of $\mathbf{1 f}(428 \mathrm{mg}, 0.978 \mathrm{mmol})$ in $\mathrm{CH}_{2} \mathrm{Cl}_{2}(2 \mathrm{~mL})$ at $0{ }^{\circ} \mathrm{C}$ was added a solution of thiobenzoic acid (152 mg, $1.10 \mathrm{mmol}$ ) in $2 \mathrm{~mL}$ of $\mathrm{CH}_{2} \mathrm{Cl}_{2}$. After stirring for $14 \mathrm{~h}$ at $\mathrm{rt}$, the solvent was evaporated and the residue was purified by $\mathrm{CC}$ to give $453 \mathrm{mg}$ (79\%) of $\mathbf{8}$ as a yellow foam. IR $\left(\mathrm{CHCl}_{3}\right): 3450 \mathrm{w}, 3020 \mathrm{w}$, $3000 m, 2940 m, 2860 w, 2840 w, 1690 s, 1680 s, 1630 w, 1600 s, 1510 m, 1485 m, 1460 m, 1430 s, 1365 m$, $1350 m, 1320 m, 1285 m, 1255 m, 1180 w, 1160 s, 1140 m, 1110 m, 1090 m, 1070 m, 1030 w, 1000 w, 975 w$, $960 w, 925 w, 885 w, 835 w .{ }^{1} \mathrm{H}-\mathrm{NMR}\left(\left(\mathrm{D}_{6}\right) \mathrm{DMSO}, 97^{\circ} \mathrm{C}\right): 7.74-7.70,7.54-7.37,7.27-7.12$ (3m, 10 arom. $\mathrm{H}, 1 \mathrm{NH})$; 6.49-6.48, 6.39-6.38 (2m, 3 arom. $\mathrm{H})$; 3.87-3.79 (m, $2 \mathrm{H}$ of $\left.-\mathrm{CH}_{2} \mathrm{NCH}_{2}-\right)$; $3.75(s, 2 \mathrm{MeO})$; $3.61(s, \mathrm{MeN})$; 3.18-3.07 ( $m, 2 \mathrm{H}$ of $\left.-\mathrm{CH}_{2} \mathrm{NCH}_{2}-\right)$; 2.56-2.39 ( $\left.m,-\mathrm{CH}_{2} \mathrm{CCH}_{2}-\right)$; $1.71\left(s, \mathrm{Me}_{2} \mathrm{C}\right)$. ${ }^{13} \mathrm{C}-\mathrm{NMR}\left(\left(\mathrm{D}_{6}\right) \mathrm{DMSO}, 97{ }^{\circ} \mathrm{C}\right): 207.8(s, \mathrm{C}=\mathrm{S}) ; 165.3(s, \mathrm{C}=\mathrm{O}) ; 160.0$ ( $s, 2$ arom. COMe); $152.8(s$, OCON); 148.7, 147.9, 134.0 (3s, 3 arom. C); 130.5, 128.7, 127.3, 126.9, 126.6, 125.1 (6d, 10 arom. CH); 102.7, 98.2 (2d, 3 arom. $\mathrm{CH}) ; 80.0$ ( $\left.s, \mathrm{Me}_{2} \mathrm{CO}\right) ; 63.1$ ( $\left.s, \mathrm{C}^{\prime} 4^{\prime}\right)$ ); 54.7 (q, $\left.2 \mathrm{MeO}\right) ; 48.6$ (q, $\left.\mathrm{MeN}\right) ; 39.3(t$, $\left.-\mathrm{CH}_{2} \mathrm{NCH}_{2}-\right) ; 35.0\left(t,-\mathrm{CH}_{2} \mathrm{CCH}_{2}-\right) ; 28.1\left(q, \mathrm{Me}_{2} \mathrm{C}\right)$. ESI-MS: $598\left(100,[M+\mathrm{Na}]^{+}\right)$.

General procedure for the synthesis of dipeptide amides 10a-d (azirine coupling). To a solution of the corresponding azirine 1 in $\mathrm{MeCN}$, THF or $\mathrm{CH}_{2} \mathrm{Cl}_{2}$ at $0{ }^{\circ} \mathrm{C}, \mathrm{Z}$ - or Fmoc-protected D-Ala was added and the mixture stirred at $\mathrm{rt}$ for $16 \mathrm{~h}$. After evapration of the solvent, the residue was purified by CC.

Z-D-Ala-Thp-N(Ph)Me (10a). The reaction of $400 \mathrm{mg}(1.85 \mathrm{mmol})$ of azirine $\mathbf{1 d}$ and $457 \mathrm{mg}(2.05$ $\mathrm{mmol})$ of Z-D-Ala-OH in MeCN (4 mL) followed by CC ( $\left.\mathrm{Et}_{2} \mathrm{O} / \mathrm{AcOEt} 1: 1\right)$ gave $746 \mathrm{mg}(92 \%)$ of $\mathbf{1 0 a}$. 
Colorless solid; mp 103-105 ${ }^{\circ} \mathrm{C}$. IR $\left(\mathrm{CHCl}_{3}\right): 3520 m, 3330 w, 3070 w, 3030 w, 3000 m, 2960 m, 2930 w$, $2860 w, 1690 s, 1640 s, 1595 m, 1500 s, 1470 w, 1450 m, 1430 w, 1370 m, 1350 m, 1320 m, 1290 m, 1230 m$, $1170 w, 1150 w, 1110 m, 1070 m, 1030 w, 1000 w, 980 w, 960 w, 910 w, 880 w, 860 w, 825 w, 700 m .{ }^{1} \mathrm{H}-\mathrm{NMR}$ $\left(\mathrm{CDCl}_{3}\right)$ : 7.37-7.29, 7.14-7.12 (2m, 10 arom. H); $5.95(s, 1 \mathrm{NH}) ; 5.19(d, J=7.1,1 \mathrm{NH}) ; 5.10,5.04(2 d$, $\left.J_{\mathrm{AB}}=12.3, \mathrm{PhCH}_{2} \mathrm{O}\right) ; 3.74-3.62,3.40-3.24\left(2 m, \mathrm{HC}(2)\right.$ of $\left.\mathrm{Ala},-\mathrm{CH}_{2} \mathrm{OCH}_{2}-\right) ; 3.21(\mathrm{~s}, \mathrm{MeN}) ; 2.33-2.25$, 1.96-1.91 (2m, $\left.-\mathrm{CH}_{2} \mathrm{CCH}_{2}-\right) ; 1.26\left(d, J=7.0\right.$, Me of Ala). ${ }^{13} \mathrm{C}-\mathrm{NMR}\left(\mathrm{CDCl}_{3}\right): 171.3,170.8(2 s, 2 \mathrm{C}=\mathrm{O})$; 156.1 ( $s$, OCON); 144.7, 135.9 (2s, 2 arom. C); 129.5, 128.5, 128.3, 128.0, 127.8, 127.2 (6d, 10 arom. $\mathrm{CH}) ; 67.2,63.0,62.9\left(3 t, \mathrm{PhCH}_{2} \mathrm{O},-\mathrm{CH}_{2} \mathrm{OCH}_{2}-\right)$; 58.1 ( $\left.s, \mathrm{C}\left(4^{\prime}\right)\right)$; 50.1 (d, $\mathrm{HC}(2)$ of Ala); $41.4(q, \mathrm{MeN})$; 33.7, $33.1\left(2 t,-\mathrm{CH}_{2} \mathrm{CCH}_{2}-\right) ; 17.2$ (q, Me of Ala). ESI-MS: $901\left(47,[2 M+\mathrm{Na}]^{+}\right), 462\left(100,[M+\mathrm{Na}]^{+}\right)$. $\left[\alpha^{21}{ }_{\mathrm{D}}\right]+20.6(\mathrm{c} 1.003)$.

Fmoc-D-Ala-Tht-N(Ph)Me (10b). The reaction of $500 \mathrm{mg}(2.15 \mathrm{mmol})$ of azirine 1e and $737 \mathrm{mg}(2.34$ mmol) of Z-D-Ala-OH in THF (5 mL) followed by CC (hexane/AcOEt 1:2) gave $1074 \mathrm{mg}(92 \%)$ of $\mathbf{1 0 b}$. Colorless solid; mp 130-132 ${ }^{\circ} \mathrm{C}$. IR $\left(\mathrm{CHCl}_{3}\right): 3420 m, 3340 m, 3000 m, 2960 m, 1690 s, 1640 s, 1595 m$, $1495 s, 1465 w, 1450 m, 1430 w, 1370 m, 1350 w, 1320 m, 1280 m, 1250 m, 1170 w, 1130 w, 1105 w, 1070 m$, 1040w, 975w. ${ }^{1} \mathrm{H}-\mathrm{NMR}\left(\mathrm{CDCl}_{3}\right): 7.76,7.56(2 d, J=7.4,4$ Fmoc-H); 7.39 ( $d, J=7.4,2$ Fmoc-H); 7.33-7.25, 7.14-7.11 (2m, 7 arom. H); $5.95(s, 1 \mathrm{NH}) ; 5.22(d, J=7.6,1 \mathrm{NH})$; 4.44-4.32 (m, Fmoc-CH $)_{2}$; $4.20(t, J=6.6$, Fmoc-C(9)H); 3.60 (quint, $J=7.2, \mathrm{HC}(2)$ of Ala); 3.21 ( $s, \mathrm{MeN}) ; 2.69-2.65,2.52-2.24$ $\left(2 m, 4 \mathrm{CH}_{2}\right) ; 1.24\left(d, J=7.1\right.$, Me of Ala). ${ }^{13} \mathrm{C}-\mathrm{NMR}\left(\mathrm{CDCl}_{3}\right): 171.7,170.8(2 s, 2 \mathrm{C}=\mathrm{O}) ; 156.2(s$, OCON); 144.9, 143.7, 143.5, 141.3 (4s, 5 arom. C); 129.5, 127.8, 127.2, 127.1, 124.8, 120.1 (6d, 13 arom. $\mathrm{CH}) ; 67.2\left(t, \mathrm{Fmoc}_{-} \mathrm{CH}_{2}\right) ; 59.7\left(s, \mathrm{C}\left(4^{\prime}\right)\right) ; 50.0$ (d, $\mathrm{HC}(2)$ of Ala); 47.0 (d, Fmoc-C(9)H); $41.5(q, \mathrm{MeN})$; 34.2, 33.7 (2t, $-\mathrm{CH}_{2} \mathrm{CCH}_{2}-$ ); 23.1, 22.9 (2t, $\left.-\mathrm{CH}_{2} \mathrm{SCH}_{2}-\right)$; 17.3 (q, Me of Ala). ESI-MS: 566 (100, $\left.[M+\mathrm{Na}]^{+}\right) \cdot\left[\alpha_{\mathrm{D}}^{21}\right]+22.2(\mathrm{c} 0.962)$.

Z-D-Ala-Pip(Ddz)-N(Ph)Me (10c). The reaction of $536 \mathrm{mg}(1.23 \mathrm{mmol})$ of azirine $\mathbf{1 f}$ and $295 \mathrm{mg}(1.36$ mmol) of Z-D-Ala-OH in $\mathrm{CH}_{2} \mathrm{Cl}_{2}(10 \mathrm{~mL})$ led to analytically pure crystalline 10c $(771 \mathrm{mg}, 95 \%)$. Colorless powder; mp 150-152 ${ }^{\circ} \mathrm{C}$. IR (KBr): 3430m, 3060w, 3000w, 2980m, 2930m, 2880w, 2830w, $1715 s, 1690 s, 1670 s, 1650 m, 1640 w, 1630 w, 1610 s, 1590 s, 1545 m, 1540 m, 1525 m, 1495 m, 1470 m$, $1455 m, 1415 m, 1380 m, 1360 m, 1315 m, 1300 m, 1285 m, 1275 m, 1255 s, 1205 m, 1155 m, 1140 m, 1100 m$, 1070m, 1050s, 1035m, 1025m, 995w, 965w, 940m, 845m, 770m, 740m, 730m, 700m. ${ }^{1} \mathrm{H}-\mathrm{NMR}$ ((D $\left.\left.{ }_{6}\right) \mathrm{DMSO}\right): 7.86(s, 1 \mathrm{NH}) ; 7.35-7.27(m, 10$ arom. H); $7.15(d, J=7.3,1 \mathrm{NH}) ; 6.34-6.42,6.38-6.36$ $(2 m, 3$ arom. $\mathrm{H}) ; 5.09,5.01\left(2 d, J_{\mathrm{AB}}=12.6, \mathrm{PhCH}_{2} \mathrm{O}\right) ; 4.02-3.50\left(m, \mathrm{HC}(2)\right.$ of Ala, $2 \mathrm{H}$ of $\left.-\mathrm{CH}_{2} \mathrm{NCH}_{2}-\right)$; $3.72(s, 2 \mathrm{MeO}) ; 3.25-2.75\left(m, 2 \mathrm{H}\right.$ of $\left.-\mathrm{CH}_{2} \mathrm{NCH}_{2}-\right) ; 3.18(s, \mathrm{MeN}) ; 2.11-1.58\left(m,-\mathrm{CH}_{2} \mathrm{CCH}_{2}-\right) ; 1.67(s$, $\left.\mathrm{Me}_{2} \mathrm{C}\right) ; 1.20$ ( $d, J=7.1$, Me of Ala). ${ }^{13} \mathrm{C}-\mathrm{NMR}\left(\left(\mathrm{D}_{6}\right) \mathrm{DMSO}\right): 171.7,171.2(2 s, 2 \mathrm{C}=\mathrm{O}) ; 160.2(s, 2$ arom. COMe); 155.6, 152.9 (2s, 2 OCON); 149.1, 145.1, 137.0 (3s, 3 arom. C); 128.9, 128.2, 127.7, 127.5, 127.2, 126.7 (6d, 10 arom. $\mathrm{CH}) ; 102.6,97.9$ (2d, 2 arom. $\mathrm{CH}) ; 80.2\left(s, \mathrm{Me}_{2} C \mathrm{O}\right) ; 65.2\left(t, \mathrm{PhCH}_{2} \mathrm{O}\right) ; 57.3$ 
$\left.\left(s, \mathrm{C}^{\prime} 4^{\prime}\right)\right) ; 55.0(q, 2 \mathrm{MeO}) ; 50.7\left(d, \mathrm{HC}(2)\right.$ of Ala); $39.9(q, \mathrm{MeN}) ; 39.7,39.2\left(2 t,-\mathrm{CH}_{2} \mathrm{NCH}_{2}-\right)$; 32.0, $31.4\left(2 t,-\mathrm{CH}_{2} \mathrm{CCH}_{2}-\right) ; 28.9,28.5\left(2 q, \mathrm{Me}_{2} \mathrm{C}\right) ; 18.3$ (q, Me of Ala). ESI-MS: $683\left(100,[M+\mathrm{Na}]^{+}\right)$. Anal. Calcd for $\mathrm{C}_{36} \mathrm{H}_{44} \mathrm{~N}_{4} \mathrm{O}_{8}$ (660.77): C 65.44, H 6.71, N 8.48. Found: C 65.33, H 6.71, N 8.31.

Fmoc-D-Ala-Pip(Ddz)-N(Ph)Me (10d). The reaction of $860 \mathrm{mg}(1.97 \mathrm{mmol})$ of azirine 1 f and $617 \mathrm{mg}$ (1.98 mmol) of Fmoc-D-Ala-OH in $\mathrm{CH}_{2} \mathrm{Cl}_{2}(10 \mathrm{~mL}$ ) followed by $\mathrm{CC}$ (hexane/AcOEt 1:2) gave $1249 \mathrm{mg}$, $85 \%$ ) of 10d. Colorless powder; mp 170-171 ${ }^{\circ} \mathrm{C}$. IR (KBr): 3440m, 3060w, 2980m, 2940m, 2880w, $2830 w, 1710 s, 1695 s, 1685 s, 1675 s, 1615 s, 1590 s, 1540 m, 1530 s, 1500 m, 1470 m, 1450 m, 1430 m, 1415 s$, $1380 m, 1365 w, 1315 m, 1300 m, 1280 m, 1235 s, 1205 m, 1195 m, 1160 m, 1130 m, 1100 m, 1070 m, 1050 m$, $1030 m, 1000 w, 980 w, 975 w, 940 m, 850 m, 770 m, 740 m, 760 m, 740 m, 700 m .{ }^{1} \mathrm{H}-\mathrm{NMR}\left(\left(\mathrm{D}_{6}\right) \mathrm{DMSO}\right): 7.85$, $7.69(2 d, J=7.4,4$ Fmoc-H); 7.44-7.14 (m, 9 arom. H, $1 \mathrm{NH}) ; 6.86(d, J=7.9,1 \mathrm{NH}) ; 6.49-6.46$, 6.40-6.36 (2m, 3 arom. H); 4.43-4.21 ( $m$, Fmoc- $\mathrm{CH}_{2}, \mathrm{HC}(2)$ of Ala); 4.04-4.34 ( $m$, Fmoc-C(9)H); 3.75 $(s, 2 \mathrm{MeO}) ; 3.74-3.50\left(m, 2 \mathrm{H}\right.$ of $\left.-\mathrm{CH}_{2} \mathrm{NCH}_{2}-\right) ; 3.20(s, \mathrm{MeN}) ; 3.25-3.07\left(m, 2 \mathrm{H}\right.$ of $\left.-\mathrm{CH}_{2} \mathrm{NCH}_{2}-\right)$; 2.15-1.97 ( $\left.m,-\mathrm{CH}_{2} \mathrm{CCH}_{2}-\right) ; 1.70\left(s, \mathrm{Me}_{2} \mathrm{C}\right) ; 1.25\left(d, J=7.0\right.$, Me of Ala). ${ }^{13} \mathrm{C}-\mathrm{NMR}\left(\left(\mathrm{D}_{6}\right) \mathrm{DMSO}\right): 172.1$, $171.7(2 s, 2 \mathrm{C}=\mathrm{O}) ; 160.6$ (s, 2 arom. COMe); 156.1, $153.4(2 s, 2 \mathrm{OCON}) ; 149.5,145.5,144.2,141.1(4 s$, 6 arom. C); 129.3, 128.0, 127.6, 127.4, 127.1, 125.7, 120.4, 103.0, 98.3 (9d, 16 arom. CH); 80.6 (s, $\left.\mathrm{Me}_{2} \mathrm{CO}\right) ; 66.0\left(t\right.$, Fmoc- $\left.C \mathrm{H}_{2}\right) ; 57.7\left(s, \mathrm{C}\left(4^{\prime}\right)\right) ; 55.4(q, 2 \mathrm{MeO}) ; 50.1,47.0$ (2d, $\mathrm{HC}(2)$ of Ala, Fmoc-C(9)H); 40.0 (q, MeN); 39.3, $38.5\left(2 t,-\mathrm{CH}_{2} \mathrm{NCH}_{2}-\right)$; 32.6, $31.9\left(2 t,-\mathrm{CH}_{2} \mathrm{CCH}_{2}-\right)$; 29.5, $28.8(2 q$, $\left.\mathrm{Me}_{2} \mathrm{C}\right) ; 18.8\left(q, \mathrm{Me}\right.$ of Ala). ESI-MS: $771\left(100,[\mathrm{M+Na}]^{+}\right)$.

General procedure for the selective methanolysis of dipeptide amides 10a-d. The corresponding dipeptide amide 10 was dissolved in $\mathrm{MeOH}$ at $0{ }^{\circ} \mathrm{C}$, and $\mathrm{HCl}$ gas was bubbled through the solution until it reached ca. $60{ }^{\circ} \mathrm{C}$. Then, the mixture was stirred for $2 \mathrm{~h}$ at $\mathrm{rt}, 1 \mathrm{~N}$ aqueous $\mathrm{HCl}$ was added, and the mixture extracted with $\mathrm{CH}_{2} \mathrm{Cl}_{2}(3 \times)$. The combined organic layer was dried with $\mathrm{MgSO}_{4}$ and the solvent evaporated in vacuo. The residue was purified by $\mathrm{CC}$ or crystallization.

Z-D-Ala-Thp-OMe (11a). The reaction of $1.020 \mathrm{~g}(2.32 \mathrm{mmol})$ of 10a in MeOH $(10 \mathrm{~mL})$ followed by CC (hexane/AcOEt 1:5) gave $775 \mathrm{mg}(92 \%)$ of 11a. Colorless foam. IR $\left(\mathrm{CHCl}_{3}\right): 3520 \mathrm{~m}, 3330 w, 3020 w$, $3000 m, 2960 m, 2860 m, 1740 s, 1700 s, 1500 s, 1465 w, 1455 m, 1435 w, 1380 w, 1350 w, 1320 m, 1305 m$, $1290 m, 1230 s, 1155 w, 1140 m, 1115 m, 1070 m, 1030 w, 1015 w, 1000 w, 910 w, 880 w, 840 w, 700 m$. ${ }^{1} \mathrm{H}-\mathrm{NMR}\left(\mathrm{CDCl}_{3}\right)$ : 7.35-7.27 (m, 5 arom. H); $6.79(s, 1 \mathrm{NH}) ; 5.36(d, J=7.3,1 \mathrm{NH}) ; 5.15,5.10\left(2 d, J_{\mathrm{AB}}=\right.$ 12.1, $\mathrm{PhCH}_{2} \mathrm{O}$ ); 4.27 (quint, $J=7.1, \mathrm{HC}(2)$ of Ala); 3.81-3.72 (m, $2 \mathrm{H}$ of $\left.-\mathrm{CH}_{2} \mathrm{OCH}_{2}-\right) ; 3.70(s, \mathrm{MeO})$; 3.62-3.48 ( $m, 2 \mathrm{H}$ of $\left.-\mathrm{CH}_{2} \mathrm{OCH}_{2}-\right)$; 2.21-2.10, 1.96-1.89 (2m, $\left.-\mathrm{CH}_{2} \mathrm{CCH}_{2}-\right)$; 1.37 (d, J= 7.1, Me of Ala). ${ }^{13} \mathrm{C}-\mathrm{NMR}\left(\mathrm{CDCl}_{3}\right): 173.2,172.0(2 s, 2 \mathrm{C}=\mathrm{O}) ; 156.4(s, \mathrm{OCON}) ; 136.0$ ( $s, 1$ arom. C); 128.6, 128.4, 128.1 (3d, 5 arom. $\mathrm{CH}) ; 67.3,63.3\left(2 t, \mathrm{PhCH}_{2} \mathrm{O},-\mathrm{CH}_{2} \mathrm{OCH}_{2}-\right) ; 56.5\left(s, \mathrm{C}\left(4^{\prime}\right)\right) ; 52.6(s, \mathrm{MeO}) ; 50.2(d, \mathrm{HC}(2)$ of Ala); 32.6, $32.5\left(2 t,-\mathrm{CH}_{2} \mathrm{CCH}_{2}-\right)$; 17.5 (q, Me of Ala). ESI-MS: $387\left(100,[\mathrm{M}+\mathrm{Na}]^{+}\right) .\left[\alpha^{21}{ }_{\mathrm{D}}\right]+22.6(\mathrm{c}$ 
1.045).

Fmoc-D-Ala-Tht-OMe (11b). The reaction of $659 \mathrm{mg}(1.21 \mathrm{mmol})$ of $\mathbf{1 0 b}$ in $\mathrm{MeOH}(5 \mathrm{~mL})$ followed by CC (hexane/AcOEt 1:2) gave $534 \mathrm{mg}(94 \%)$ of 11b. Colorless foam. IR $\left(\mathrm{CHCl}_{3}\right): 3430 \mathrm{~m}, 3340 \mathrm{w}, 3060 \mathrm{w}$, 3010m, 2950m, 1735s, 1695s, 1505s, 1465w, 1450m, 1435m, 1380w, 1350w, 1320m, 1280m, 1220s, $1110 w, 1075 m, 1060 m, 1020 w, 930 w, 710 m, 670 m .{ }^{1} \mathrm{H}-\mathrm{NMR}\left(\mathrm{CDCl}_{3}\right): 7.75,7.57(2 d, J=7.4,4$ Fmoc-H); 7.39, $7.29(2 t, J=7.4,4$ Fmoc-H); $6.77(s, 1 \mathrm{NH}) ; 5.50(d, J=7.7,1 \mathrm{NH}) ; 4.40(d, J=6.7$, Fmoc- $\left.\mathrm{CH}_{2}\right) ; 4.35-4.27$ ( $m, \mathrm{HC}(2)$ of Ala); $4.20(t, J=6.8$, Fmoc-C(9)H); $3.68(s, \mathrm{MeO}) ; 2.74-2.63$, 2.55-2.45, 2.33-2.15 (3m, $\left.4 \mathrm{CH}_{2}\right) ; 1.37\left(d, J=6.9\right.$, Me of Ala). ${ }^{13} \mathrm{C}-\mathrm{NMR}\left(\mathrm{CDCl}_{3}\right): 173.4,171.9(2 s, 2$ $\mathrm{C}=\mathrm{O}) ; 156.4(s, \mathrm{OCON}) ; 143.6,141.3$ (2s, 4 arom. C); 127.8, 127.1, 125.0, 120.1 (4d, 8 arom. CH); 67.3 $\left(t, \mathrm{Fmoc}-\mathrm{CH}_{2}\right) ; 58.1$ ( $\left.s, \mathrm{C}\left(4^{\prime}\right)\right) ; 52.5$ ( $\left.q, \mathrm{MeO}\right) ; 50.3$ (d, HC(2) of Ala); 47.0 (d, Fmoc-C(9)H); $33.3(t$, $\left.-\mathrm{CH}_{2} \mathrm{CCH}_{2}-\right)$; 23.5, $23.4\left(2 t,-\mathrm{CH}_{2} \mathrm{SCH}_{2}-\right) ; 18.0$ (q, Me of Ala). ESI-MS: $491\left(100,[M+\mathrm{Na}]^{+}\right) .\left[\alpha^{21}{ }_{\mathrm{D}}\right]$ +12.1 (c 0.962).

Z-D-Ala-Pip-OMe (11c). A solution of $1.742 \mathrm{~g}(2.64 \mathrm{mmol})$ of 10c in $\mathrm{MeOH}(20 \mathrm{~mL})$ was reacted with $\mathrm{HCl}$ gas. Then, the solvent was evaporated under vacuum, the residue was dissolved in $\mathrm{CH}_{2} \mathrm{Cl}_{2}$ and washed with $1 \mathrm{~N}$ aqueous $\mathrm{NaOH}$. The aqueous phase was extracted with $\mathrm{CH}_{2} \mathrm{Cl}_{2}$, the combined organic phase was dried $\left(\mathrm{MgSO}_{4}\right)$ and the solvent evaporated. $\mathrm{CC}\left(\mathrm{Et}_{2} \mathrm{O} / \mathrm{AcOEt} 1: 4\right)$ gave $650 \mathrm{mg}(68 \%)$ of 11c. Colorless foam. ${ }^{1} \mathrm{H}-\mathrm{NMR}\left(\mathrm{CDCl}_{3}\right)$ : 7.35-7.25 (m, 5 arom. H, $\left.1 \mathrm{NH}\right) ; 5.70$ (d-like, $\left.1 \mathrm{NH}\right) ; 5.11$ (br. $s$, $\mathrm{PhCH}_{2} \mathrm{O}$ ); 4.35-4.25 ( $m, \mathrm{HC}(2)$ of Ala); 3.75 (br. $\left.s, 1 \mathrm{NH}\right) ; 3.67$ ( $\left.s, \mathrm{MeO}\right) ; 2.99-2.70\left(m,-\mathrm{CH}_{2} \mathrm{NCH}_{2}-\right)$; 2.10-2.00 ( $\left.m,-\mathrm{CH}_{2} \mathrm{CCH}_{2}-\right) ; 1.38\left(d, J=7.0\right.$, Me of Ala). ${ }^{13} \mathrm{C}-\mathrm{NMR}\left(\mathrm{CDCl}_{3}\right): 173.5,172.2(2 s, 2 \mathrm{C}=\mathrm{O})$; 156.2 ( $s, \mathrm{OCON}) ; 136.3$ ( $s, 1$ arom. C); 128.5, $128.2,128.0$ (3d, 5 arom. CH); $67.0\left(t, \mathrm{PhCH}_{2} \mathrm{O}\right) ; 57.2(s$, $\left.\mathrm{C}\left(4^{\prime}\right)\right) ; 52.5$ (q, $\left.\mathrm{MeO}\right) ; 47.1$ (d, $\mathrm{HC}(2)$ of $\left.\mathrm{Ala}\right) ; 41.5\left(t,-\mathrm{CH}_{2} \mathrm{NCH}_{2}-\right) ; 32.0\left(t,-\mathrm{CH}_{2} \mathrm{CCH}_{2}-\right) ; 18.2(q, \mathrm{Me}$ of Ala). CI-MS: $364\left(100,[M+1]^{+}\right), 256(32)$.

Z-D-Ala-Pip(Fmoc)-OMe (11d). A solution of $1.846 \mathrm{~g}(2.79 \mathrm{mmol})$ of 10c in $\mathrm{MeOH}(20 \mathrm{~mL})$ was reacted with $\mathrm{HCl}$ gas. Then, the solvent was evaporated under vacuum, the residue was dissolved in $\mathrm{CH}_{2} \mathrm{Cl}_{2}(20 \mathrm{~mL})$, cooled to $0{ }^{\circ} \mathrm{C}$, and $1.56 \mathrm{~mL}(11.2 \mathrm{mmol}) \mathrm{Et}_{3} \mathrm{~N}$ and $1.446 \mathrm{~g}$ (5.59 mmol) of Fmoc-chloride were added. After stirring for $14 \mathrm{~h}$ at $\mathrm{rt}$, the mixture was washed with $1 \mathrm{~N} \mathrm{HCl}$. The aqueous phase was extracted with $\mathrm{CH}_{2} \mathrm{Cl}_{2}$, the combined organic phase was dried $\left(\mathrm{MgSO}_{4}\right)$ and the solvent evaporated. CC (hexane/AcOEt 1:2) gave $1.236 \mathrm{~g}$ (76\%) of 11d. Colorless foam. IR $\left(\mathrm{CHCl}_{3}\right)$ : $3430 w, 3070 w, 3000 w, 2960 w, 2870 w, 1740 m, 1695 s, 1500 m, 1480 m, 1450 m, 1435 m, 1350 w, 1335 w$, $1280 m, 1270 m, 1240 m, 1205 m, 1155 m, 1135 w, 1095 w, 1065 m, 1050 m, 1040 w, 1030 w, 925 w .{ }^{1} \mathrm{H}-\mathrm{NMR}$ $\left(\mathrm{CDCl}_{3}\right): 7.67,7.47$ (2d, $J=7.4,4$ Fmoc-H); $7.31(t, J=7.4,2$ Fmoc-H); 7.25-7.17 ( $m, 7$ arom. H); 6.69 $(s, 1 \mathrm{NH}) ; 5.24(d, J=7.1,1 \mathrm{NH}) ; 5.05,4.09\left(2 d, J_{\mathrm{AB}}=12.2, \mathrm{PhCH}_{2} \mathrm{O}\right) ; 4.40-4.32,4.17-4.12,3.83-3.57$ (3m, Fmoc- $\mathrm{CH}_{2}$, Fmoc-C(9)H, $\mathrm{HC}(2)$ of Ala, $2 \mathrm{H}$ of $\left.-\mathrm{CH}_{2} \mathrm{NCH}_{2}-\right)$; $3.60(s, \mathrm{MeO}) ; 2.99-2.89(m, 2 \mathrm{H}$ of $\left.-\mathrm{CH}_{2} \mathrm{NCH}_{2}-\right) ; 1.86-1.79\left(m,-\mathrm{CH}_{2} \mathrm{CCH}_{2}-\right) ; 1.27\left(d, J=7.0, \mathrm{Me}\right.$ of Ala). ${ }^{13} \mathrm{C}-\mathrm{NMR}\left(\mathrm{CDCl}_{3}\right): 172.9,172.0$ 
$(2 s, 2 \mathrm{C}=\mathrm{O}) ; 156.3,154.8(2 s, 2 \mathrm{OCON}) ; 143.9,141.3,135.9(3 s, 5$ arom. C); 128.5, 128.3, 128.0, 127.6, 127.0, 124.8, 119.9 (7d, 13 arom. CH); $67.2\left(t, \mathrm{PhCH}_{2} \mathrm{O}, \mathrm{Fmoc}-\mathrm{CH}_{2}\right) ; 57.2\left(s, \mathrm{C}\left(4^{\prime}\right)\right)$; 52.5 (q, MeO); 50.3, $47.3\left(2 d, \mathrm{HC}(2)\right.$ of Ala, Fmoc-C(9)H); $39.5\left(t,-\mathrm{CH}_{2} \mathrm{NCH}_{2}-\right) ; 31.7\left(t,-\mathrm{CH}_{2} \mathrm{CCH}_{2}-\right)$; 17.3 (q, Me of Ala). ESI-MS: $608\left(100,[M+N a]^{+}\right) .\left[\alpha_{D}^{21}\right]+10.4$ (c 1.024).

The same product was obtained from $11 \mathrm{c}(578 \mathrm{mg}, 1.59 \mathrm{mmol})$ in $\mathrm{CH}_{2} \mathrm{Cl}_{2}(10 \mathrm{~mL})$ at $0{ }^{\circ} \mathrm{C}$ by treatment with $270 \mu \mathrm{L}(1.94 \mathrm{mmol}) \mathrm{Et}_{3} \mathrm{~N}$ and Fmoc-chloride $(450 \mathrm{mg}, 1.74 \mathrm{mmol})$. After stirring for $14 \mathrm{~h}$ at $\mathrm{rt}$ and purification by CC (hexane/AcOEt 1:2), $811 \mathrm{mg}(87 \%)$ of 11d were isolated.

Fmoc-D-Ala-Pip(Z)-OMe (11f). A solution of $1.371 \mathrm{~g}(1.83 \mathrm{mmol})$ of $\mathbf{1 0 d}$ in $\mathrm{MeOH}(15 \mathrm{~mL})$ was reacted with $\mathrm{HCl}$ gas. Then, the solvent was evaporated under vacuum, the residue was dissolved in $\mathrm{CH}_{2} \mathrm{Cl}_{2}(15 \mathrm{~mL})$, cooled to $0{ }^{\circ} \mathrm{C}$, and $1.10 \mathrm{~mL}(7.9 \mathrm{mmol}) \mathrm{Et}_{3} \mathrm{~N}$ and $624 \mathrm{mg}(3.66 \mathrm{mmol})$ of Z-chloride were added. After stirring for $14 \mathrm{~h}$ at $\mathrm{rt}$, the mixture was washed with $1 \mathrm{~N} \mathrm{HCl}$. The aqueous phase was extracted with $\mathrm{CH}_{2} \mathrm{Cl}_{2}$, the combined organic phase was dried $\left(\mathrm{MgSO}_{4}\right)$ and the solvent evaporated. $\mathrm{CC}$ (hexane/AcOEt 1:2) gave $882 \mathrm{~g}(76 \%)$ of 11f. Colorless foam. IR $\left(\mathrm{CHCl}_{3}\right)$ : 3430w, 3330w, 3060w, 3000m, $2960 w, 2870 w, 1740 m, 1690 s, 1510 m, 1500 m, 1480 w, 1450 m, 1430 m, 1385 w, 1365 w, 1355 w, 1320 m$, $1280 m, 1265 m, 1230 m, 1150 w, 1135 w, 1075 m, 1020 w, 1000 w, 975 w, 925 w .{ }^{1} \mathrm{H}-\mathrm{NMR}\left(\mathrm{CDCl}_{3}\right): 7.68$, $7.47(2 d, J=7.4,4$ Fmoc-H); 7.33-7.17 ( $m, 9$ arom. H); $6.68(s, 1 \mathrm{NH}) ; 5.30(d, J=7.5,1 \mathrm{NH}) ; 5.03(s$, $\left.\mathrm{PhCH}_{2} \mathrm{O}\right) ; 4.32\left(d, J=6.8\right.$, Fmoc- $\left.\mathrm{CH}_{2}\right) ; 4.19-4.08$ ( $m, \mathrm{HC}(2)$ of Ala); $4.11(t, J=6.8$, Fmoc-C(9)H); 3.83-3.70 ( $m, 2 \mathrm{H}$ of $\left.-\mathrm{CH}_{2} \mathrm{NCH}_{2}-\right)$; $3.60(s, \mathrm{MeO})$; 3.13-3.01 ( $m, 2 \mathrm{H}$ of $\left.-\mathrm{CH}_{2} \mathrm{NCH}_{2}-\right)$; $1.98-1.89(m$, $\left.-\mathrm{CH}_{2} \mathrm{CCH}_{2}-\right) ; 1.27\left(d, J=7.0\right.$, Me of Ala). ${ }^{13} \mathrm{C}-\mathrm{NMR}\left(\mathrm{CDCl}_{3}\right): 173.0,172.1(2 s, 2 \mathrm{C}=\mathrm{O}) ; 156.2,155.0(2 s$, 2 OCON); 143.5, 141.2, 136.5 (3s, 5 arom. C); 128.4, 128.0, 127.8, 127.7, 127.0, 124.8, 120.0 (7d, 13 arom. $\mathrm{CH}) ; 67.2\left(t, \mathrm{PhCH}_{2} \mathrm{O}, \mathrm{Fmoc}-\mathrm{CH}_{2}\right) ; 57.3$ ( $\left.s, \mathrm{C}\left(4^{\prime}\right)\right) ; 52.5$ (q, MeO); 50.2, 47.0 (2d, $\mathrm{HC}(2)$ of Ala, Fmoc-C(9)H); $39.5\left(t,-\mathrm{CH}_{2} \mathrm{NCH}_{2}-\right)$; 31.9, 31.7 (2t, $\left.-\mathrm{CH}_{2} \mathrm{CCH}_{2}-\right)$; 17.8 ( $q$, Me of Ala). ESI-MS: 608 (100, $[M+\mathrm{Na}]^{+}$). Anal. Calcd for $\mathrm{C}_{33} \mathrm{H}_{35} \mathrm{~N}_{3} \mathrm{O}_{7}$ (585.66): C 67.68, H 6.02, N 7.17. Found: C 67.19, H 6.09, N 7.07. $\left[\alpha^{21}\right]+12.1(\mathrm{c} 1.061)$.

Synthesis of dipeptide Z-D-Ala-Pip(Boc)-OMe (11e). To a solution of 11d (1.183 g, $1.97 \mathrm{mmol})$ in $\mathrm{CH}_{2} \mathrm{Cl}_{2}(5 \mathrm{~mL})$ was added piperidine $(0.5 \mathrm{~mL})$. The mixture was stirred at $\mathrm{rt}$ for $1 \mathrm{~h}$, the solvent evaporated, the residue dissolved in $\mathrm{CH}_{2} \mathrm{Cl}_{2}$, and $860 \mathrm{mg}(3.94 \mathrm{mmol})$ Boc-anhydride and $550 \mu \mathrm{L}$ (3.94 mmol) $\mathrm{Et}_{3} \mathrm{~N}$ were added. After stirring for $14 \mathrm{~h}$ at $\mathrm{rt}$, the solvent was evaporated and the residue purified by CC (hexane/AcOEt 1:2) yielding $643 \mathrm{mg}(70 \%)$ of 11e. Colorless foam. IR $\left(\mathrm{CHCl}_{3}\right): 3430 \mathrm{~m}, 3340 \mathrm{w}$, $3060 w, 3000 m, 2980 m, 1730 s, 1680 s, 1510 m, 1500 s, 1450 m, 1430 m, 1390 m, 1370 m, 1320 m, 1280 s$, $1240 s, 1170 m, 1150 m, 1095 w, 1065 m, 1030 w, 1010 w, 975 w, 930 w, 860 w .{ }^{1} \mathrm{H}-\mathrm{NMR}\left(\mathrm{CDCl}_{3}\right): 7.34-7.29$ $\left(m, 5\right.$ arom. H); $6.86(s, 1 \mathrm{NH}) ; 5.46(d, J=7.5,1 \mathrm{NH}) ; 5.12,5.07\left(2 d, J_{\mathrm{AB}}=12.2, \mathrm{PhCH}_{2} \mathrm{O}\right) ; 4.29-4.20$, 3.85-3.71 (2m, $\mathrm{HC}(2)$ of Ala, $2 \mathrm{H}$ of $\left.-\mathrm{CH}_{2} \mathrm{NCH}_{2}-\right)$; $3.67(s, \mathrm{MeO}) ; 3.04-2.91\left(m, 2 \mathrm{H}\right.$ of $\left.-\mathrm{CH}_{2} \mathrm{NCH}_{2}-\right)$; 
2.02-1.85 ( $\left.m,-\mathrm{CH}_{2} \mathrm{CCH}_{2}-\right) ; 1.46\left(s, \mathrm{Me}_{3} \mathrm{C}\right) ; 1.36$ (d, $J=7.0$, Me of Ala). ${ }^{13} \mathrm{C}-\mathrm{NMR}\left(\mathrm{CDCl}_{3}\right): 173.2$, 172.1 (2s, $2 \mathrm{C}=\mathrm{O})$ ) 156.2, 154.3 (2s, 2 OCON); 135.9 (s, 1 arom. C); 128.5, 128.2, 127.9 (3d, 5 arom. $\mathrm{CH}) ; 79.7\left(s, \mathrm{Me}_{3} C\right) ; 67.1\left(t, \mathrm{PhCH}_{2} \mathrm{O}\right) ; 57.3\left(s, \mathrm{C}\left(4^{\prime}\right)\right) ; 52.4(q, \mathrm{MeO}) ; 50.2(d, \mathrm{HC}(2)$ of Ala); $39.1(t$, $\left.-\mathrm{CH}_{2} \mathrm{NCH}_{2}-\right)$; 31.9, $31.6\left(2 t,-\mathrm{CH}_{2} \mathrm{CCH}_{2}-\right) ; 28.3$ ( $\left.q, M e_{3} \mathrm{C}\right) ; 17.6$ ( $q$, Me of Ala). ESI-MS: 486 (100, $\left.[M+\mathrm{Na}]^{+}\right)$. Anal. Calcd for $\mathrm{C}_{23} \mathrm{H}_{33} \mathrm{~N}_{3} \mathrm{O}_{7}$ (463.53): C 59.60, H 7.18, N 9.07. Found: C 59.33, H 7.29, N 8.76. $\left[\alpha^{21}\right]+11.5$ (c 0.989$)$.

\section{Selective deprotection of the terminal amino group of dipeptide esters $11 a, b$.}

H-D-Ala-Thp-OMe (12a). To a solution of dipeptide 11 (167 $\mathrm{mg}, 0.458 \mathrm{mmol})$ in $\mathrm{MeOH}(3 \mathrm{~mL})$ was added $16.7 \mathrm{mg}$ of $\mathrm{Pd} / \mathrm{C}(10 \%)$. This mixture was stirred under an $\mathrm{H}_{2}$-atmosphere at $\mathrm{rt}$ until disappearance of 11a (TLC). After filtration via Celite, the solvent of the filtrate was evaporated and the residue dried in $\mathrm{HV}$ : $85 \mathrm{mg}(81 \%)$ of 12a. White powder. ${ }^{1} \mathrm{H}-\mathrm{NMR}\left(\mathrm{CF}_{3} \mathrm{CO}_{2} \mathrm{D}\right): 4.52(q, J=7.0, \mathrm{HC}(2)$ of Ala); 4.39-4.31, 4.18-3.99 (2m, $\left.-\mathrm{CH}_{2} \mathrm{OCH}_{2}-\right)$; 3.45 (s, $\left.\mathrm{MeO}\right) ; 2.62-2.53,2.06-1.98\left(2 m,-\mathrm{CH}_{2} \mathrm{CCH}_{2}-\right) ; 1.70(d$, $J=7.0$, Me of Ala). ${ }^{13} \mathrm{C}-\mathrm{NMR}\left(\mathrm{CF}_{3} \mathrm{CO}_{2} \mathrm{D}\right): 172.8,171.7(2 s, 2 \mathrm{C}=\mathrm{O}) ; 62.1\left(t,-\mathrm{CH}_{2} \mathrm{OCH}_{2}-\right) ; 55.6(s$, $\left.\mathrm{C}\left(4^{\prime}\right)\right)$; 54.0 ( $\left.s, \mathrm{MeO}\right) ; 50.3$ ( $d, \mathrm{HC}(2)$ of Ala); 34.6, $33.5\left(2 t,-\mathrm{CH}_{2} \mathrm{CCH}_{2}-\right)$; 18.2 (q, Me of Ala). CI-MS: $461\left(35,[2 M+1]^{+}\right), 231\left(100,[M+1]^{+}\right)$.

H-D-Ala-Tht-OMe (12b). The dipeptide 11b (120 mg, $0.256 \mathrm{mmol})$ was dissolved in piperidine (3 $\mathrm{mL})$ at rt. After $10 \mathrm{~min}$, the precipitate was filtered and washed with AcOEt to give $48 \mathrm{mg}(76 \%)$ of $\mathbf{1 2 b}$. White powder. ${ }^{1} \mathrm{H}-\mathrm{NMR}\left(\mathrm{CF}_{3} \mathrm{CO}_{2} \mathrm{D}\right): 4.50(q, J=7.0, \mathrm{HC}(2)$ of Ala); $3.41(s, \mathrm{MeO}) ; 2.63-2.55$, 2.23-2.18, 2.02-1.95, 1.87-1.79 (4m, $\left.4 \mathrm{CH}_{2}\right) ; 1.68$ ( $d, J=7.0$, Me of Ala). ${ }^{13} \mathrm{C}-\mathrm{NMR}\left(\mathrm{CF}_{3} \mathrm{CO}_{2} \mathrm{D}\right): 174.8$, $174.3(2 s, 2 \mathrm{C}=\mathrm{O}) ; 60.0\left(s, \mathrm{C}\left(4^{\prime}\right)\right) ; 52.6(s, \mathrm{MeO}) ; 48.4$ (d, $\mathrm{HC}(2)$ of Ala); 37.8, $36.6\left(2 t,-\mathrm{CH}_{2} \mathrm{CCH}_{2}-\right)$; 24.4, $23.4\left(2 t,-\mathrm{CH}_{2} \mathrm{SCH}_{2}-\right) ; 20.8\left(q\right.$, Me of Ala). CI-MS: $461\left(35,[2 M+1]^{+}\right), 231\left(100,[M+1]^{+}\right)$.

\section{Synthesis of tripeptides 14 via coupling of dipeptides with aspartic acid derivatives.}

Z-Asp(OBn)-D-Ala-Thp-OMe (14a). To a solution of 11a (764 mg, $2.10 \mathrm{mmol})$ in $\mathrm{MeOH}$ (10 mL)/DMF (15 mL) was added $\mathrm{Pd} / \mathrm{C}(10 \%, 38 \mathrm{mg})$ and the mixture was treated with $\mathrm{H}_{2}$ until the disappearance of 11a (TLC). The catalyst was removed by filtration via Celite and $\mathrm{MeOH}$ was evaporated. To the remaining solution were added Z-Asp(OBn)-OH (13a, 900 mg, 2.52 mmol), PyBOP (1.312 g, 2.52 $\mathrm{mmol})$, and $\mathrm{Et}_{3} \mathrm{~N}(700 \mu \mathrm{L}, 3 \mathrm{mmol})$, and the mixture was stirred for $16 \mathrm{~h}$ at $\mathrm{rt}$. Evaporation of the solvent, $\mathrm{CC}\left(\mathrm{Et}_{2} \mathrm{O} / \mathrm{AcOEt} 1: 2\right)$, and crystallization from hexane/AcOEt/MeOH yielded $956 \mathrm{mg}(80 \%)$ 14a. Colorless crystals; mp $127-128{ }^{\circ} \mathrm{C}$. IR $\left(\mathrm{CHCl}_{3}\right): 3420 m, 3070 w, 3030 m, 3000 m, 2960 m, 2860 w, 1760 s$, $1715 s, 1695 s, 1680 s, 1515 s, 1505 s, 1455 m, 1445 m, 1430 w, 1405 w, 1390 m, 1355 m, 1290 m, 1260 m$, $1190 m, 1160 m, 1145 m, 1105 m, 1075 w, 1050 m, 1030 w, 1000 w, 980 w, 970 w, 960 w, 920 w, 845 w$. ${ }^{1} \mathrm{H}-\mathrm{NMR}\left(\mathrm{CDCl}_{3}\right)$ : 7.28-7.21 (m, 10 arom. $\left.\mathrm{H}\right) ; 6.92-6.88(m, 2 \mathrm{NH}) ; 5.93(d, J=8.4,1 \mathrm{NH}) ; 5.07-4.97$ 
$\left(m, 2 \mathrm{PhCH}_{2} \mathrm{O}\right) ; 4.55-4.48$ ( $m, \mathrm{HC}(2)$ of Asp); 4.40 (quint, $J=7.3, \mathrm{HC}(2)$ of Ala); 3.70-3.63, 3.56-3.51 $\left(2 m,-\mathrm{CH}_{2} \mathrm{OCH}_{2}-\right)$; 3.61 ( $\left.s, \mathrm{MeO}\right) ; 3.05-2.70$ ( $m, \mathrm{CH}_{2}$ of Asp); 2.11-1.85 (m, $\left.-\mathrm{CH}_{2} \mathrm{CCH}_{2}-\right) ; 1.27(d, J=$ 7.0, Me of Ala). ${ }^{13} \mathrm{C}-\mathrm{NMR}\left(\mathrm{CDCl}_{3}\right): 173.3,171.5,170.7(3 s, 3 \mathrm{C}=\mathrm{O}) ; 156.1$ ( $\left.s, \mathrm{OCON}\right) ; 135.6,135.0(2 s$, 2 arom. C); 128.6, 128.4, 128.1, 128.0 (4d, 10 arom. CH); 67.5, 66.9 (2t, $\left.2 \mathrm{PhCH}_{2} \mathrm{O}\right)$; 63.3, 63.2 (2t, $\left.-\mathrm{CH}_{2} \mathrm{OCH}_{2}-\right)$; $56.6\left(s, \mathrm{C}\left(4^{\prime}\right)\right) ; 52.4(s, \mathrm{MeO}) ; 51.4,49.0\left(2 d, \mathrm{HC}(2)\right.$ of Ala, $\mathrm{HC}(2)$ of Asp); $35.9\left(t, \mathrm{CH}_{2}\right.$ of Asp); 32.6, $32.2\left(2 t,-\mathrm{CH}_{2} \mathrm{CCH}_{2}-\right)$; 16.8 (q, Me of Ala). ESI-MS: $592\left(100,[M+\mathrm{Na}]^{+}\right) .\left[\alpha_{\mathrm{D}}^{21}\right]+8.7(\mathrm{c}$ 0.946).

Fmoc-Asp(OtBu)-D-Ala-Tht-OMe (14b). To a solution of 11b (262 mg, $0.559 \mathrm{mmol})$ in DMSO (3 mL) was added piperidine (49 $\mathrm{mg}, 0.575 \mathrm{mmol}$ ) at $\mathrm{rt}$. After $40 \mathrm{~min}$, the residual piperidine was removed by distillation. Then, $232 \mathrm{mg}(0.564 \mathrm{mmol})$ Fmoc-Asp(OtBu)-OH (13b) and $303 \mathrm{mg}(0.583 \mathrm{mmol})$ PyBOP were added and the mixture stirred for $16 \mathrm{~h}$ at rt. The solvent was removed by distillation (high vacuum) and the residue purified by $\mathrm{CC}$ (hexane/AcOEt 1:3) to give $330 \mathrm{mg}$ (92\%) of 14b. Colorless foam. IR $\left(\mathrm{CHCl}_{3}\right): 3420 w, 3020 w, 3000 m, 2980 m, 2950 w, 1730 s, 1715 s, 1680 s, 1505 s, 1465 w, 1450 m, 1435 m$, $1420 w, 1405 w, 1395 w, 1370 m, 1315 m, 1280 m, 1260 m, 1155 w, 1105 w, 1080 w, 1065 m, 1050 m, 1020 w$, $930 w, 920 w, 900 w, 880 w, 855 w, 840 w, 820 w .{ }^{1} \mathrm{H}-\mathrm{NMR}\left(\mathrm{CDCl}_{3}\right): 7.76,7.57(2 d, J=7.4,4 \mathrm{Fmoc}-\mathrm{H}) ; 7.40$ $(t, J=7.4,2$ Fmoc-H); 7.34-7.30 (m, 2 Fmoc-H); $6.88(d, J=7.6,1 \mathrm{NH}) ; 6.75(s, 1 \mathrm{NH}) ; 5.88(d$-like, br, $1 \mathrm{NH}$ ); 4.51-4.43, 4.24-4.19 (2m, Fmoc-CH $\mathrm{CH}_{2}$ Fmoc-C(9)H, HC(2) of Asp, HC(2) of Ala); 3.68 ( $\left.s, \mathrm{MeO}\right)$; 2.92-2.18 ( $m, \mathrm{CH}_{2}$ of Asp, $\left.4 \mathrm{CH}_{2}\right) ; 1.45\left(s, \mathrm{Me}_{3} \mathrm{C}\right) ; 1.35\left(d, J=7.0\right.$, Me of Ala). ${ }^{13} \mathrm{C}-\mathrm{NMR}\left(\mathrm{CDCl}_{3}\right): 173.4$, 171.2, 170.9, $170.6(4 s, 4 \mathrm{C}=\mathrm{O}) ; 156.1$ ( $s, \mathrm{OCON}) ; 143.5,141.2(2 s, 4$ arom. C); 127.7, 127.0, 124.8, 120.0 (4d, 8 arom. $\mathrm{CH}) ; 82.0\left(s, \mathrm{Me}_{3} C\right)$; 67.3 ( $\left.t, \mathrm{Fmoc}_{-} \mathrm{CH}_{2}\right) ; 58.1$ (s, C(4')); 52.4 (q, MeO); 51.4, 48.9, $47.0\left(3 d, \mathrm{HC}(2)\right.$ of Ala, $\mathrm{HC}(2)$ of Asp, Fmoc-C(9)H); 37.1, 33.3, $33.2\left(3 t, \mathrm{CH}_{2}\right.$ of Asp, $\left.-\mathrm{CH}_{2} \mathrm{CCH}_{2}-\right)$; $28.0\left(q, \mathrm{Me}_{3} \mathrm{C}\right) ; 23.4\left(t,-\mathrm{CH}_{2} \mathrm{SCH}_{2}-\right) ; 16.8\left(q\right.$, Me of Ala). ESI-MS: $662\left(100,[M+\mathrm{Na}]^{+}\right), 440(47$, $\left.[M-\mathrm{Fmoc}+\mathrm{Na}]^{+}\right) \cdot\left[\alpha_{\mathrm{D}}^{21}\right]+5.2(\mathrm{c} 1.085)$.

Z-Asp(OBn)-D-Ala-Pip(Boc)-OMe (14c). To a solution of 11e (545 mg, $1.18 \mathrm{mmol})$ in $\mathrm{MeOH}(10$ $\mathrm{mL}) / \mathrm{DMF}(15 \mathrm{~mL})$ was added $\mathrm{Pd} / \mathrm{C}(10 \%, 47 \mathrm{mg})$ and the mixture was treated with $\mathrm{H}_{2}$ until the disappearence of 11e (TLC). The catalyst was removed by filtration via Celite and $\mathrm{MeOH}$ was evaporated. To the remaining solution were added Z-Asp(OBn)-OH (13a, 505 mg, $1.41 \mathrm{mmol}$ ), PyBOP (730 mg, 1.40 $\mathrm{mmol})$, and $\mathrm{Et}_{3} \mathrm{~N}(200 \mu \mathrm{L}, 1.4 \mathrm{mmol})$, and the mixture was stirred for $16 \mathrm{~h}$ at $\mathrm{rt}$. Evaporation of the solvent and subsequent $\mathrm{CC}\left(\mathrm{Et}_{2} \mathrm{O} / \mathrm{AcOEt} 1: 1\right)$ yielded $657 \mathrm{mg}(84 \%)$ 14c. Colorless foam. IR $\left(\mathrm{CHCl}_{3}\right)$ : $3420 m, 3060 w, 3020 m, 3000 m, 2970 m, 1740 s, 1715 s, 1680 s, 1515 m, 1500 m, 1495 m, 1450 m, 1430 m$, $1390 m, 1365 m, 1355 m, 1280 m, 1270 m, 1240 m, 1170 m, 1150 m, 1070 m, 1050 w, 975 w, 920 w, 855 w, 845 w$. ${ }^{1} \mathrm{H}-\mathrm{NMR}\left(\mathrm{CDCl}_{3}\right)$ : 7.35-7.26 ( $m, 10$ arom. $\left.\mathrm{H}\right) ; 6.92(s, 1 \mathrm{NH}) ; 6.87(d, J=7.3,1 \mathrm{NH}) ; 5.93(d, J=9.0,1$ $\mathrm{NH}$ ); 5.15-5.02 ( $\left.m, 2 \mathrm{PhCH}_{2} \mathrm{O}\right)$; 4.60-4.54 ( $m, \mathrm{HC}(2)$ of Asp); 4.45 (quint, $J=7.3$, $\mathrm{HC}(2)$ of Ala); $3.82-3.75\left(m, 2 \mathrm{H}\right.$ of $\left.-\mathrm{CH}_{2} \mathrm{NCH}_{2}-\right) ; 3.67(s, \mathrm{MeO}) ; 3.16-3.03,2.84-2.76\left(2 m, 2 \mathrm{H}\right.$ of $-\mathrm{CH}_{2} \mathrm{NCH}_{2}-, \mathrm{CH}_{2}$ 
of Asp); 2.04-1.96 ( $\left.m,-\mathrm{CH}_{2} \mathrm{CCH}_{2}-\right) ; 1.42\left(s, \mathrm{Me}_{3} \mathrm{C}\right) ; 1.32\left(d, J=7.0\right.$, Me of Ala). ${ }^{13} \mathrm{C}-\mathrm{NMR}\left(\mathrm{CDCl}_{3}\right)$ : 173.4, 171.7, 171.6, 170.8 (4s, 4 C=O); 156.2, 154.6 (2s, 2 OCON); 135.7, 135.1 (2s, 2 arom. C); 128.7, 128.5, 128.2, 127.9 (4d, 10 arom. $\mathrm{CH}) ; 79.8\left(s, \mathrm{Me}_{3} C\right)$; 67.6, $\left.67.0\left(2 t, 2 \mathrm{PhCH}_{2} \mathrm{O}\right) ; 57.6\left(s, \mathrm{C}^{\prime} 4^{\prime}\right)\right) ; 52.5(q$, $\mathrm{MeO}) ; 51.4,49.1$ (2d, $\mathrm{HC}(2)$ of Ala, $\mathrm{HC}(2)$ of Asp); 39.3, 35.9 (2t, $-\mathrm{CH}_{2} \mathrm{NCH}_{2}-, \mathrm{CH}_{2}$ of Asp); 32.0, 31.6 $\left(2 t,-\mathrm{CH}_{2} \mathrm{CCH}_{2}-\right) ; 28.4\left(q, \mathrm{Me}_{3} \mathrm{C}\right) ; 16.9$ ( $q$, Me of Ala). ESI-MS: $691\left(100,[M+\mathrm{Na}]^{+}\right) .\left[\alpha_{\mathrm{D}}^{21}\right]+7.8(\mathrm{c}$ 0.992).

Fmoc-Asp(OtBu)-D-Ala-Pip(Z)-OMe (14d). To a solution of $11 f$ (250 mg, $0.472 \mathrm{mmol})$ in DMSO (4 $\mathrm{mL}$ ) was added piperidine ( $45 \mu \mathrm{L}, 0.456 \mathrm{mmol})$ at $\mathrm{rt}$. After $40 \mathrm{~min}$, the residual piperidine was removed by distillation. Then, $194 \mathrm{mg}(0.472 \mathrm{mmol})$ Fmoc-Asp(OtBu)-OH (13b) and $245 \mathrm{mg}(0.471 \mathrm{mmol})$ PyBOP were added and the mixture stirred for $16 \mathrm{~h}$ at $\mathrm{rt}$. The solvent was removed by distillation (high vacuum) and the residue purified by $\mathrm{CC}$ (hexane/AcOEt 1:3) to give $147 \mathrm{mg}$ (44\%) of 14d. Colorless foam. IR $\left(\mathrm{CHCl}_{3}\right): 3450 w, 3330 w, 3060 w, 3020 w, 3000 m, 2980 m, 2950 w, 1730 s, 1710 s, 1690 s, 1515 s$, 1505 , 1450m, 1430m, 1395w, 1370m, 1320m, 1280m, 1255m, 1245m, 1150m, 1105w, 1060m, 1020w, $955 w, 925 w, 900 w, 855 w, 840 w, 820 w .{ }^{1} \mathrm{H}-\mathrm{NMR}\left(\mathrm{CDCl}_{3}\right): 7.75,7.56(2 d, J=7.4,4$ Fmoc-H); 7.42-7.21 $\left(m, 9\right.$ arom. H); $6.97(s, 1 \mathrm{NH}) ; 6.89(d, J=7.6,1 \mathrm{NH}) ; 5.81(d$-like, $1 \mathrm{NH}) ; 5.10\left(s, \mathrm{PhCH}_{2} \mathrm{O}\right) ; 4.50-4.41$ $\left(m, \mathrm{HC}(2)\right.$ of Asp, $\mathrm{HC}(2)$ of Ala, Fmoc- $\left.\mathrm{CH}_{2}\right) ; 4.21(t, J=6.6$, Fmoc-C(9)H); 3.89-3.73 ( $m, 2 \mathrm{H}$ of $\left.-\mathrm{CH}_{2} \mathrm{NCH}_{2}-\right) ; 3.67$ ( $\left.s, \mathrm{MeO}\right) ; 3.27-3.16\left(m, 2 \mathrm{H}\right.$ of $\left.-\mathrm{CH}_{2} \mathrm{NCH}_{2}-\right) ; 2.98-2.87,2.74-2.66\left(2 m, \mathrm{CH}_{2}\right.$ of Asp); 2.07-1.98 ( $\left.m,-\mathrm{CH}_{2} \mathrm{CCH}_{2}-\right)$; $1.41\left(s, \mathrm{Me}_{3} \mathrm{C}\right) ; 1.35\left(d, J=7.1\right.$, Me of Ala). ${ }^{13} \mathrm{C}-\mathrm{NMR}\left(\mathrm{CDCl}_{3}\right): 173.1$, 171.6, 170.7 (3s, $4 \mathrm{C}=\mathrm{O})$; 156.1, 155.0 (2s, 2 OCON); 143.5, 143.4, 141.2, 136.6 (4s, 5 arom. C); 128.4, 127.9, 127.8, 127.5, 127.0, 124.8, 120.0 (7d, 13 arom. CH); 82.0 ( $\left.s, \mathrm{Me}_{3} C\right)$; 67.2, $67.1\left(2 t, \mathrm{PhCH}_{2} \mathrm{O}\right.$, Fmoc- $\left.\mathrm{CH}_{2}\right) ; 57.4\left(s, \mathrm{C}\left(4^{\prime}\right)\right)$; $52.4(q, \mathrm{MeO}) ; 51.4,48.9,47.0(3 d, \mathrm{HC}(2)$ of Ala, $\mathrm{HC}(2)$ of Asp, Fmoc-C(9)H); 39.5, $37.0\left(2 t,-\mathrm{CH}_{2} \mathrm{NCH}_{2}-, \mathrm{CH}_{2}\right.$ of Asp); 32.0, $31.6\left(2 t,-\mathrm{CH}_{2} \mathrm{CCH}_{2}-\right)$; $27.9\left(q, M e_{3} \mathrm{C}\right)$; $16.9\left(q\right.$, Me of Ala). ESI-MS: $779\left(100,[M+\mathrm{Na}]^{+}\right) .\left[\alpha^{21}{ }_{\mathrm{D}}\right]+5.1(\mathrm{c} 1.097)$.

\section{Preparation of the zwitterionic tripeptides $15 \mathrm{a}-\mathrm{d}$.}

H-Asp-D-Ala-Thp-OMe (15a). To a solution of 14a (125 mg, $0.219 \mathrm{mmol})$ in $\mathrm{MeOH}(10 \mathrm{~mL})$ was added $\mathrm{Pd} / \mathrm{C}(10 \%, 15 \mathrm{mg})$ and the mixture was treated with $\mathrm{H}_{2}$ for $6 \mathrm{~h}$. The catalyst was removed by filtration via Celite, $\mathrm{MeOH}$ was evaporated, and the residue dried (HV) to give $89 \mathrm{mg}$ (98\%) of 15a. Colorless powder; mp $129-131^{\circ} \mathrm{C}$. IR (KBr): $3460 m, 3300 m, 3060 m, 2960 m, 1745 m, 1730 m, 1695 s, 1680 s, 1665 s$, $1645 m, 1630 m, 1580 m, 1575 m, 1565 s, 1550 s, 1540 s, 1505 w, 1495 w, 1470 m, 1450 m, 1445 m, 1430 m$, $1400 m, 1390 m, 1385 m, 1345 w, 1335 w, 1310 w, 1290 m, 1260 m, 1230 m, 1150 m, 1100 m, 1070 m, 1030 w$, $1015 w, 1005 w, 985 w, 965 w, 920 w, 890 w, 870 w, 840 w .{ }^{1} \mathrm{H}-\mathrm{NMR}\left(\mathrm{CD}_{3} \mathrm{OD}\right): 4.43-4.35$ ( $m, \mathrm{HC}(2)$ of Asp); 4.09-404 ( $m, \mathrm{HC}(2)$ of Ala); 3.79-3.65 ( $\left.m,-\mathrm{CH}_{2} \mathrm{OCH}_{2}-\right)$; 3.68 ( $\left.s, \mathrm{MeO}\right) ; 2.71-2.56$ ( $m, \mathrm{CH}_{2}$ of Asp); 2.17-1.89 ( $\left.m,-\mathrm{CH}_{2} \mathrm{CCH}_{2}-\right) ; 1.37\left(d, J=7.1\right.$, Me of Ala). ${ }^{13} \mathrm{C}-\mathrm{NMR}\left(\mathrm{CD}_{3} \mathrm{OD}\right): 174.6,173.6,173.4,168.6$ 
$(4 s, 4 \mathrm{C}=\mathrm{O}) ; 62.2,62.8\left(2 t,-\mathrm{CH}_{2} \mathrm{OCH}_{2}-\right) ; 56.4\left(s, \mathrm{C}\left(4^{\prime}\right)\right) ; 51.4(s, \mathrm{MeO}) ; 50.8,48.9$ (2d, $\mathrm{HC}(2)$ of Ala, $\mathrm{HC}(2)$ of Asp); 36.7 ( $t, \mathrm{CH}_{2}$ of Asp); 32.2, 31.6 (2t, $\left.-\mathrm{CH}_{2} \mathrm{CCH}_{2}-\right)$; 16.6 (q, Me of Ala). ESI-MS: 384 (27, $\left.[M+\mathrm{K}]^{+}\right), 368\left(23,[M+\mathrm{Na}]^{+}\right), 346\left(100,[M+1]^{+}\right) \cdot\left[\alpha_{\mathrm{D}}^{21}\right]+31.3(\mathrm{c} 1.020)$.

H-Asp-D-Ala-Tht-OMe (15b). To a solution of $50 \mathrm{mg}(0.078 \mathrm{mmol}) \mathbf{1 4 b}$ in $\mathrm{CH}_{2} \mathrm{Cl}_{2}(1 \mathrm{~mL})$ was added piperidine $(100 \mu \mathrm{L}, 1.01 \mathrm{mmol})$ and the mixture stirred for $30 \mathrm{~min}$ at $\mathrm{rt}$. Then, the solvent and piperidine were evaporated and the residue purified by $\mathrm{CC}\left(\mathrm{CH}_{2} \mathrm{Cl}_{2} / \mathrm{MeOH} / \mathrm{NH}_{3}(\mathrm{aq})\right.$ 100:2.5:0.25) yielding $30 \mathrm{mg}$ (92\%) of $\mathrm{H}$-Asp $(\mathrm{OtBu})$-D-Ala-Tht-OMe as a colorless foam. IR $\left(\mathrm{CHCl}_{3}\right): 3440 \mathrm{~m}, 3020 \mathrm{w}, 2980 \mathrm{~m}, 2950 \mathrm{w}$, $1740 s, 1730 s, 1715 s, 1695 s, 1680 s, 1660 s, 1650 s, 1510 s, 1495 m, 1480 m, 1395 w, 1370 m, 1335 w, 1330 w$, $1320 w, 1280 s, 1260 m, 1150 s, 1085 w, 1060 w, 1015 w, 950 w, 940 w, 930 w, 840 w .{ }^{1} \mathrm{H}-\mathrm{NMR}\left(\mathrm{CDCl}_{3}\right): 7.94$ $(d, J=7.1,1 \mathrm{NH}) ; 7.21(s, 1 \mathrm{NH}) ; 4.45$ (quint, $J=7.0, \mathrm{HC}(2)$ of Ala); $3.64(s, \mathrm{MeO}) ; 3.64-3.57(m$, $\mathrm{HC}(2)$ of Asp); 2.79-2.64, 2.55-2.43, 2.30-2.05 (3m, $\mathrm{CH}_{2}$ of Asp, $\left.4 \mathrm{CH}_{2}\right) ; 1.89\left(s, \mathrm{NH}_{2}\right) ; 1.39\left(s, \mathrm{Me}_{3} \mathrm{C}\right)$; $1.35\left(d, J=7.0\right.$, Me of Ala). ${ }^{13} \mathrm{C}-\mathrm{NMR}\left(\mathrm{CDCl}_{3}\right): 173.3,172.6,170.7,169.8(4 s, 4 \mathrm{C}=\mathrm{O}) ; 80.4\left(s, \mathrm{Me}_{3} C\right)$; $57.1\left(s, \mathrm{C}\left(4^{\prime}\right)\right) ; 52.4$ ( $\left.q, \mathrm{MeO}\right) ; 50.9,47.5$ (2d, $\mathrm{HC}(2)$ of Ala, $\mathrm{HC}(2)$ of Asp); $40.2\left(t, \mathrm{CH}_{2}\right.$ of Asp); $32.2(t$, $\left.-\mathrm{CH}_{2} \mathrm{CCH}_{2}-\right)$; 27.1 (q, Me $\left.e_{3} \mathrm{C}\right) ; 22.5,22.4$ (2t, $\left.-\mathrm{CH}_{2} \mathrm{SCH}_{2}-\right)$; 15.9 (q, Me of Ala). ESI-MS: 440 (38, $\left.[M+\mathrm{Na}]^{+}\right), 418\left(100,[M+1]^{+}\right) .\left[\alpha^{21}{ }_{\mathrm{D}}\right]+21.1(\mathrm{c} 1.010)$. A solution of $85 \mathrm{mg}(0.203 \mathrm{mmol})$ of the product in TFA ( $1 \mathrm{~mL})$ was stirred for $2 \mathrm{~h}$. After evaporation of TFA and drying of the residue (HV), ca. $100 \mathrm{mg}$ of 15b, still containing TFA, were obtained. ${ }^{1} \mathrm{H}-\mathrm{NMR}\left(\mathrm{CD}_{3} \mathrm{OD}\right): 4.48-4.40,4.23-4.17$ (2 m, HC(2) of Asp, $\mathrm{HC}(2)$ of Ala); $3.67(s, \mathrm{MeO})$; 3.05-2.75, 2.55-2.08 (2m, $\mathrm{CH}_{2}$ of Asp, $\left.4 \mathrm{CH}_{2}\right) ; 1.37(d, J=7.1$, Me of Ala). ${ }^{13} \mathrm{C}-\mathrm{NMR}\left(\mathrm{CD}_{3} \mathrm{OD}\right)$ : 175.4, 174.4, 172.9, 169.0 (4s, $\left.4 \mathrm{C}=\mathrm{O}\right) ; 59.7$ ( $s, \mathrm{C}\left(4^{\prime}\right)$ ); 51.4 ( $q$, MeO); 49.3, 49.0 (2d, $\mathrm{HC}(2)$ of Ala, $\mathrm{HC}(2)$ of Asp); 36.0, 34.9, 34.2 (3t, $\mathrm{CH}_{2}$ of Asp, $\left.-\mathrm{CH}_{2} \mathrm{CCH}_{2}-\right)$; 24.3, $24.2(2 t$, $\left.-\mathrm{CH}_{2} \mathrm{SCH}_{2}-\right) ; 17.8$ ( $q$, Me of Ala).

H-Asp-D-Ala-Pip(Boc)-OMe (15c). To a solution of 14c $(252 \mathrm{mg}, 0.377 \mathrm{mmol})$ in $\mathrm{MeOH}(10 \mathrm{~mL})$ was added $\mathrm{Pd} / \mathrm{C}(10 \%, 35 \mathrm{mg})$ and the mixture was treated with $\mathrm{H}_{2}$ for $7 \mathrm{~h}$. The catalyst was removed by filtration via Celite, $\mathrm{MeOH}$ was evaporated, and the residue dried (HV) to give $167 \mathrm{mg}(100 \%)$ of 15c. Colorless powder; mp 137-140 ${ }^{\circ} \mathrm{C}$. IR (KBr): 3400m, 3250m, 3060m, 2985m, 1740m, 1730m, 1690s, $1680 \mathrm{~s}, 1670 \mathrm{~s}, 1635 \mathrm{~m}, 1615 \mathrm{~m}, 1570 \mathrm{~m}, 1550 \mathrm{~m}, 1540 \mathrm{~m}, 1515 \mathrm{~m}, 1505 \mathrm{~m}, 1485 \mathrm{~m}, 1470 \mathrm{~m}, 1465 \mathrm{~m}, 1455 \mathrm{~m}$, $1445 m, 1430 s, 1390 m, 1370 m, 1330 w, 1280 m, 1240 m, 1170 m, 1150 m, 1090 w, 1070 m, 1010 w, 975 w$, 920w, 850w, 810w. ${ }^{1} \mathrm{H}-\mathrm{NMR}\left(\mathrm{CD}_{3} \mathrm{OD}\right)$ : 4.40-4.33 ( $m, \mathrm{HC}(2)$ of Asp); 4.10-4.04 ( $m, \mathrm{HC}(2)$ of Ala), 3.83-3.74 ( $m, 2 \mathrm{H}$ of $\left.-\mathrm{CH}_{2} \mathrm{NCH}_{2}-\right)$; $3.67(s, \mathrm{MeO}) ; 3.26-3.12\left(m, 2 \mathrm{H}\right.$ of $\left.-\mathrm{CH}_{2} \mathrm{NCH}_{2}-\right)$; $2.74-2.54(m$, $\mathrm{CH}_{2}$ of Asp); $2.12-1.89\left(m,-\mathrm{CH}_{2} \mathrm{CCH}_{2}-\right) ; 1.46\left(s, \mathrm{Me}_{3} \mathrm{C}\right) ; 1.37$ (d, $J=7.1$, Me of Ala). ${ }^{13} \mathrm{C}-\mathrm{NMR}$ $\left(\mathrm{CD}_{3} \mathrm{OD}\right)$ : 176.0, 175.1, 170.4, $\left.170.1(4 s, 4 \mathrm{C}=\mathrm{O}) ; 156.4(s, \mathrm{OCON}) ; 81.3\left(s, \mathrm{Me}_{3} C\right) ; 58.7\left(s, \mathrm{C}^{\prime} 4^{\prime}\right)\right) ; 53.0$ $(q, \mathrm{MeO}) ; 52.3,50.5\left(2 d, \mathrm{HC}(2)\right.$ of Ala, $\mathrm{HC}(2)$ of Asp); 41.2, $40.6\left(2 t,-\mathrm{CH}_{2} \mathrm{NCH}_{2}-\right) ; 38.2\left(t, \mathrm{CH}_{2}\right.$ of Asp); 33.1, $32.3\left(2 t,-\mathrm{CH}_{2} \mathrm{CCH}_{2}-\right)$; 28.7 ( $\left.q, \mathrm{Me}_{3} \mathrm{C}\right)$; 18.0 (q, Me of Ala). ESI-MS: $467\left(54,[M+\mathrm{Na}]^{+}\right), 445$ $\left(100,[M+1]^{+}\right) \cdot\left[\alpha^{21}\right]+18.8(\mathrm{c} 1.020)$. 
H-Asp-D-Ala-Pip(Z)-OMe (15d). To a solution of $797 \mathrm{mg}(1.05 \mathrm{mmol}) \mathbf{1 4 d}$ in $\mathrm{CH}_{2} \mathrm{Cl}_{2}(5 \mathrm{~mL})$ was added piperidine $(5 \mathrm{~mL})$ and the mixture stirred for $30 \mathrm{~min}$ at $\mathrm{rt}$. Then, the solvent and piperidine were evaporated and the residue purified by $\mathrm{CC}\left(\mathrm{CH}_{2} \mathrm{Cl}_{2} / \mathrm{MeOH} / \mathrm{NH}_{3}(\mathrm{aq})\right.$ 100:3:0.3) yielding $515 \mathrm{mg}(91 \%)$ of H-Asp $(\mathrm{OtBu})$-D-Ala-Pip(Z)-OMe as a colorless foam. IR $\left(\mathrm{CHCl}_{3}\right): 3360 m, 3060 w, 3000 m, 2980 m, 2950 w$, $1740 m, 1730 m, 1710 m, 1705 m, 1690 s, 1680 s, 1520 m, 1505 m, 1470 w, 1450 m, 1435 m, 1395 w, 1370 m$, $1330 w, 1280 m, 1245 m, 1150 m, 1095 m, 1070 m, 1020 w, 970 w, 925 w, 890 w, 850 w . ~{ }^{1} \mathrm{H}-\mathrm{NMR}\left(\mathrm{CDCl}_{3}\right)$ : $7.86(d, J=8.0,1 \mathrm{NH}) ; 7.38-7.28(m, 5$ arom. $\mathrm{H}) ; 7.23(s, 1 \mathrm{NH}) ; 5.11\left(s, \mathrm{PhCH}_{2} \mathrm{O}\right) ; 4.55-4.44(m$, $\mathrm{HC}(2)$ of Ala); 3.90-3.78 ( $m, 2 \mathrm{H}$ of $\left.-\mathrm{CH}_{2} \mathrm{NCH}_{2}-\right)$; 3.71 ( $\left.s, \mathrm{MeO}\right) ; 3.62-3.58$ ( $m, \mathrm{HC}(2)$ of Asp); 3.35-3.20 ( $m, 2 \mathrm{H}$ of $\left.-\mathrm{CH}_{2} \mathrm{NCH}_{2}-\right) ; 2.87-2.65$ ( $m, \mathrm{CH}_{2}$ of Asp); 2.08-2.01 ( $\left.m,-\mathrm{CH}_{2} \mathrm{CCH}_{2}-\right) ; 1.86(s$, $\left.\mathrm{NH}_{2}\right) ; 1.40\left(s, \mathrm{Me}_{3} \mathrm{C}\right) ; 1.38\left(d, J=7.2\right.$, Me of Ala). ${ }^{13} \mathrm{C}-\mathrm{NMR}\left(\mathrm{CDCl}_{3}\right): 174.0,173.3,172.1,171.3(4 s, 4$ $\mathrm{C}=\mathrm{O}) ; 155.1$ ( $s, \mathrm{OCON}) ; 136.7$ ( $s, 1$ arom. C); 128.5, $128.0,127.9$ (3d, 5 arom. CH); $81.6\left(s, \mathrm{Me}_{3} C\right) ; 67.2$ $\left(t, \mathrm{PhCH}_{2} \mathrm{O}\right) ; 57.4\left(s, \mathrm{C}\left(4^{\prime}\right)\right) ; 52.5$ ( $\left.q, \mathrm{MeO}\right) ; 51.6,48.5$ (2d, $\mathrm{HC}(2)$ of Ala, $\mathrm{HC}(2)$ of Asp); 40.2, 39.7 (2t, $-\mathrm{CH}_{2} \mathrm{NCH}_{2}-, \mathrm{CH}_{2}$ of Asp); $31.8\left(t,-\mathrm{CH}_{2} \mathrm{CCH}_{2}-\right) ; 28.1$ ( $\left.q, M e_{3} \mathrm{C}\right) ; 16.8$ (q, Me of Ala). ESI-MS: 557 (22, $\left.[M+\mathrm{Na}]^{+}\right), 535\left(100,[M+1]^{+}\right) \cdot\left[\alpha_{\mathrm{D}}^{21}\right]+22.3(\mathrm{c} 0.990)$. A solution of $67 \mathrm{mg}(0.125 \mathrm{mmol})$ of the product in TFA (1 mL) was stirred for $1.5 \mathrm{~h}$, whereby the color of the solution turned to pale brown. After evaporation of TFA and drying of the residue (HV), it was dissolved in $\mathrm{MeOH}$. Evaporation of the solvents and drying in $\mathrm{HV}$ yielded $82 \mathrm{mg}$ of $\mathbf{1 5 d}$, which contained still TFA. ${ }^{1} \mathrm{H}-\mathrm{NMR}\left(\mathrm{CD}_{3} \mathrm{OD}\right)$ : 7.35-7.29 ( $m, 5$ arom. H); $5.11\left(s, \mathrm{PhCH}_{2} \mathrm{O}\right)$; 4.41-4.33, 4.21-4.16 (2m, $\mathrm{HC}(2)$ of Asp, $\mathrm{HC}(2)$ of Ala); 3.88-3.82 (m, $2 \mathrm{H}$ of $\left.-\mathrm{CH}_{2} \mathrm{NCH}_{2}-\right) ; 3.66(s, \mathrm{MeO}) ; 3.31-3.20\left(m, 2 \mathrm{H}\right.$ of $\left.-\mathrm{CH}_{2} \mathrm{NCH}_{2}-\right) ; 2.99-2.82(m$, $\mathrm{CH}_{2}$ of Asp); 2.15-1.93 ( $\left.m,-\mathrm{CH}_{2} \mathrm{CCH}_{2}-\right) ; 1.37$ (d,J=7.1, Me of Ala). ${ }^{13} \mathrm{C}-\mathrm{NMR}\left(\mathrm{CD}_{3} \mathrm{OD}\right): 173.5,173.4$, 171.1, $167.4(4 s, 4 \mathrm{C}=\mathrm{O}) ; 155.3$ ( $s, \mathrm{OCON}) ; 136.5$ ( $s, 1$ arom. C); 128.1, 127.7, 127.4 (3d, 5 arom. $\mathrm{CH})$; $66.9\left(t, \mathrm{PhCH}_{2} \mathrm{O}\right) ; 57.2$ ( $\left.\left.s, \mathrm{C}^{\prime} 4^{\prime}\right)\right)$; 51.4 ( $\left.q, \mathrm{MeO}\right) ; 49.4,49.1$ (2d, $\mathrm{HC}(2)$ of Ala, $\mathrm{HC}(2)$ of Asp); 39.3, 39.2 $\left(2 t,-\mathrm{CH}_{2} \mathrm{NCH}_{2}-\right) ; 34.4$ ( $t, \mathrm{CH}_{2}$ of Asp); 31.5, 30.7 (2t, $\left.-\mathrm{CH}_{2} \mathrm{CCH}_{2}-\right)$; 16.5 (q, Me of Ala). ESI-MS: 501 $\left(13,[M+\mathrm{Na}]^{+}\right), 479\left(100,[M+1]^{+}\right)$.

\section{ACKNOWLEDGEMENTS}

We thank the Swiss National Science Foundation, the Stiftung für wissenschaftliche Forschung an der Universität Zürich, and F. Hoffmann-La Roche AG, Basel, for financial support.

\section{REFERENCES AND NOTES}

1. Part of the PhD Thesis of C.S., University of Zurich.

2. a) H. Heimgartner, Israel J. Chem., 1986, 27, 3; b) D. Obrecht and H. Heimgartner, Helv. Chim. Acta, 1987, 70, 102; c) P. Wipf and H. Heimgartner, Helv. Chim. Acta, 1988, 71, 140; d) H. Heimgartner, Angew. Chem., Int. Ed. Engl., 1991, 30, 238. 
3. a) Peptaibiotics, ed. by C. Toniolo and H. Brückner, Wiley-VHCA, Weinheim, 2009; b) N. Stoppacher, N. K. N. Neumann, L. Burgstaller, S. Zeilinger, T. Degenkolb, H. Brückner, and R. Schuhmacher, Chem. Biodivers., 2013, 10, 734; c) C. De la Fuente-Núñez, L. Whitmore, and B. A. Wallace, Peptaibols, in 'Handbook of Biologically Active Peptides', ed. by A. Kastin, Academic Press, Cambridge, 2013, pp. 150-156; d) C. Adam, A. D. Peters, M. G. Lizio, G. F. S. Whitehead, V. Diemer, J. A. Cooper, S. L. Cockroft, J. Clayden, and S. J. Webb, Chem. Eur. J., 2018, 24, 2249.

4. a) P. Wipf and H. Heimgartner, Helv. Chim. Acta, 1990, 73, 13; b) N. Pradeille and H. Heimgartner, J. Pept. Sci., 2003, 9, 827; c) R. T. N. Luykx, A. Linden, and H. Heimgartner, Helv. Chim. Acta, 2003, 86, 4093; d) N. Pradeille, O. Zerbe, K. Möhle, A. Linden, and H. Heimgartner, Chem. Biodivers., 2005, 2, 1127; e) W. Altherr, A. Linden, and H. Heimgartner, Chem. Biodivers., 2007, 4, 1144; f) N. Pradeille, M. Tzouros, K. Möhle, A. Linden, and H. Heimgartner, Chem. Biodivers., 2012, 9, 2528; g) P. Blaser, W. Altherr, A. Linden, and H. Heimgartner, Chem. Biodivers., 2013, 10, 920.

5. a) M. Hatanaka and T. Ishimaru, Bull. Chem. Soc. Jpn., 1973, 46, 2515; b) A. W. Coulter, J. B. Lombardini, and J. R. Sufrin, Mol. Pharmacol., 1974, 10, 319; c) Y. Morimoto and K. Achiwa, Chem. Pharm. Bull., 1987, 35, 3845; d) D. M. Walker and E. W. Logusch, Tetrahedron Lett., 1989, 30, 1181; e) O. Mamoun, H. Benhaoua, R. Danion-Bougot, and D. Danion, Synth. Commun., 1995, 25, 1295; f) K. Lavrador, D. Guillerm, and G. Guillerm, Bioorg. Med. Chem. Lett., 1998, 8, 1629; g) G. Schäfer and J. W. Bode, Org. Lett., 2014, 16, 1526; h) M. Caruso, E. Gatto, A. Palleschi, M. Scarselli, M. De Crescenzi, F. Formaggio, E. Longo, C. Toniolo, K. Wright, and M. Venanzi, Z. Phys. Chem., 2016, 230, 1351.

6. a) M. M. Federici and F. J. Lotspeich, Biochem. Pharmacol., 1979, 28, 1689; b) C. L. Wysong, T. S. Yokum, G. A. Morales, R. L. Gundry, M. L. McLaughlin, and R. P. Hammer, J. Org. Chem., 1996, 61, 7650; c) L. G. J. Hammarstroem, Y. Fu, S. Vail, R. P. Hammer, and M. L. McLaughlin, Org. Synth., 2005, 81, 213; d) S. Malaquin, M. Jida, J.-C. Gesquiere, R. Deprez-Poulin, and B. Deprez, Tetrahedron Lett., 2010, 51, 2983; e) J. I. Cho, M. Tanaka, S. Sato, K. Kinbara, and T. Aida, J. Am. Chem. Soc., 2010, 132, 13176; f) T. Boddaert, J. Solà, M. Helliwell, and J. Clayden, Chem. Commun., 2012, 48, 3397.

7. a) S. Stamm, A. Linden, and H. Heimgartner, Helv. Chim. Acta, 2003, 86, 1371; b) C. Strässler and H. Heimgartner, Helv. Chim. Acta, 1997, 80, 1528; c) J. L. Räber, S. A. Stoykova, C. Strässler, and H. Heimgartner, Phosphorus, Sulfur Silicon Relat. Elem., 2013, 188, 441.

8. C. Strässler, A. Linden, and H. Heimgartner, Heterocycles, 2018, 97, 333.

9. M. Rodriguez, J. M. Bland, J. W. Tsang, and M. Goodman, J. Med. Chem., 1985, 28, 1527; see also T. Yamazaki, E. Benedetti, D. Kent, and M. Goodman, Angew. Chem., Int. Ed. Engl., 1994, 33, 
1437.

10. C. Birr, W. Lochinger, G. Stahnke, and P. Lang, Liebigs Ann. Chem., 1972, 763, 162.

11. a) J. M. Villalgordo and H. Heimgartner, Helv. Chim. Acta, 1992, 75, 1866; b) J. M. Villalgordo and H. Heimgartner, Tetrahedron, 1993, 49, 7215. 